\title{
Light-scattering study of the liquid-glass transition in propylene carbonate
}

\author{
W. M. Du, G. Li, and H. Z. Cummins \\ Department of Physics, City College of the City University of New York, New York, New York 10031 \\ M. Fuchs \\ Department of Physics, Technical University of Munich, D-9646 Garching, Germany \\ J. Toulouse and L. A. Knauss \\ Department of Physics, Lehigh University, Bethlehem, Pennsylvania 18015
}

(Received 16 November 1993)

\begin{abstract}
Depolarized light-scattering spectra of propylene carbonate were obtained in the frequency range 0.2 $\mathrm{GHz}-4 \mathrm{THz}$ at temperatures from 350 to $135 \mathrm{~K}$. Analysis of the resulting susceptibility spectra revealed reasonable agreement with the predictions of the idealized mode coupling theory, yielding critical exponents $a=0.29, b=0.50$, and an exponent parameter $\lambda=0.78 \pm 0.05$. A scaling analysis demonstrated critical slowing down of the scaling frequencies from both above and below $T_{C}$, with the scaling frequency extrapolating to zero at $T_{C}=187 \pm 5 \mathrm{~K}$. The $\alpha$-relaxation peak was fit to a Kohlrausch function, which gave $\beta=0.77 \pm 0.05$ for $210 \leq T \leq 350 \mathrm{~K}$ with no sign of systematic temperature dependence. An extended mode coupling theory analysis was also carried out which corroborated the results obtained with the idealized version. Polarized Brillouin spectra were also obtained, and were analyzed with a generalized hydrodynamics approach using low-frequency sound velocity values determined separately in an ultrasonics experiment. The results indicate that the Cole-Davidson function is not an adequate model for the structural relaxation due to the neglect of $\beta$-relaxation processes, so that this analysis cannot provide a meaningful estimate of $T_{C}$. Furthermore, the $\alpha$-relaxation time associated with Brillouin scattering was found to be about five times shorter than that probed by depolarized light scattering.
\end{abstract}

PACS number(s): 64.70.Pf, 78.35. +c, 83.50.Fc, 61.20.Lc

\section{INTRODUCTION}

The dynamics of supercooled liquids approaching the liquid-glass transition has been the subject of intense study in recent years. The development of the mode coupling theory (MCT) [1,2] led to detailed predictions for the functional form and scaling properties of the correlation functions of density fluctuations $\rho_{q}(t)$ and their associated susceptibility spectra $\chi^{\prime \prime}(\omega)$, many of which have been verified by experiments. Although many of the crucial experiments have utilized neutron scattering techniques, it has also been found recently that depolarized large-angle light-scattering spectroscopy provides a powerful approach to the study of supercooled liquids, and can reveal the full form of the susceptibility spectra described by MCT.

Two recent papers have demonstrated this technique, for $\mathrm{CaKNO}_{3}$ (CKN) [3] and salol [4]. In these experiments, depolarized light-scattering spectra obtained with several settings of a tandem Fabry-Pérot interferometer were combined with data obtained with a grating spectrometer to achieve composite spectra spanning more than four decades in frequency. These composite susceptibility spectra exhibited the functional form and scaling properties predicted for the intermediate $\beta$-relaxation region by MCT for both materials, with critical slowing down of the scaling frequency as $T_{C}$ is approached from either above or below. Recently, these data have been reanalyzed using the extended version of MCT which in- cludes activated transport terms that restore ergodicity below $T_{C}$, yielding improved fits of the data at low frequencies at temperatures near to and below $T_{C}$ [5], where fits with the idealized MCT produced systematic discrepancies. A related study of orthoterphenyl (OTP) has recently been reported by Steffen et al. [6] who extended the spectral window to much lower frequencies.

The liquid-glass transition in propylene carbonate (PC) has been studied by incoherent neutron scattering by Borjesson and co-workers [7,8]. Power-law fits of the $\alpha$ relaxation time determined a crossover temperature $T_{C}=188 \mathrm{~K}$, while the temperature dependence of the nonergodicity parameter $f_{q}(T)$ gave $T_{C}=210 \mathrm{~K}$. Brillouin scattering in PC has been studied in a series of experiments by Torell and co-workers $[8-10]$ who found a cusp in the nonergodicity parameter $f_{q \rightarrow 0}(T)$ at $270 \mathrm{~K}$ [10], well above the melting temperature $T_{m}$, raising the possibility that MCT may not provide a consistent description of supercooled liquid dynamics.

Masood et al. [11] studied the sound velocity, shear viscosity, and specific heat of $\mathrm{PC}$ and three related compounds. Several dielectric studies of PC have been reported, with the results mostly analyzed using the ColeDavidson or Cole-Cole distributions [12-15,11,16-18]. The temperature-dependent viscosity of PC [19] is extremely non-Arrhenius-like, falling close to the fragile limit in Angell's classification [20] so that PC should be an appropriate material for testing the predictions of MCT. 
In the present paper, we report a depolarized lightscattering study of PC which we have analyzed using both the idealized MCT (a preliminary report of this work was presented in Ref. [21]) and the extended MCT. We have also performed polarized Brillouin scattering experiments and an ultrasonic study of PC to further explore the aspects of the dynamics studied by Elmroth, Borjesson, and Torell [10]. In Sec. II we describe the depolarized light-scattering experiments, followed in Sec. III by a brief summary of the relevant predictions of the idealized MCT. In Sec. IV we present the idealized MCT analysis of the data for the $\beta$-relaxation and $\alpha$-relaxation regimes, followed in Sec. V by the extended MCT analysis. The polarized Brillouin scattering and ultrasonics experiments are described and analyzed separately in Sec. VI. Our summary and conclusions are given in Sec. VII.

\section{EXPERIMENT}

Propylene carbonate (4-methyl-1,3-diaxolan-2-one, $\mathrm{C}_{4} \mathrm{H}_{6} \mathrm{O}_{3}$, or PC; $T_{g}=160 \mathrm{~K}, T_{m}=218 \mathrm{~K}$ ) is a high permittivity $\left(\epsilon_{0}>60\right)$ polar cyclic carbonate which supercools easily [13]. PC of $99+\%$ purity with water content less than $0.005 \%$ was purchased from Aldrich Chemical. PC is hygroscopic, and absorbed water will freeze below $0{ }^{\circ} \mathrm{C}$ producing small ice particles which strongly scatter light. The material was therefore handled in a dry nitrogen environment. It was first dried with a type $4 \AA$ molecular sieve for $24 \mathrm{~h}$. Subsequently, the dried material was transferred through a $0.1 \mu \mathrm{m}$ MSI (Micron Separations Inc.) microfilter into a cylindrical glass sample cell $1.6 \mathrm{~cm}$ in diameter by $1.5 \mathrm{~cm}$ high and flame sealed under vacuum.

The filled glass sample cell was placed in a copper sample holder with optical windows and attached to the cold finger of an Oxford ND1754 cryostat. The temperature was regulated by an Oxford ITC4 temperature controller with an accuracy of $\pm 0.1 \mathrm{~K}$. An additional thermistor located at the bottom of the sample holder close to the sample cell was used to monitor the approach of the sample temperature to equilibrium. Spectra were recorded from 350 down to $135 \mathrm{~K}$ in $10 \mathrm{~K}$ steps. To avoid crystallization for temperatures between $T_{m}$ and $T_{g}$, after each spectrum was collected the sample was reheated above $T_{m}$ and then cooled to the next desired temperature. Data were collected fifteen minutes after the sample reached thermal equilibrium.

Depolarized interferometric and Raman spectra were collected in the VH geometry at a scattering angle of $\theta=172^{\circ}$. The VH geometry and large scattering angle effectively eliminate the longitudinal and transverse acoustic modes respectively, allowing the weaker scattering due to orientational dynamics and second-order dipole-induced-dipole (DID) scattering to be observed [3]. The large scattering angle also increases the effective scattering volume, leading to enhancement of the signalto-noise ratio. Interferometric spectra were obtained with a six-pass Sandercock tandem Fabry-Pérot interferometer with finesse $\sim 50$, at four different free spectral ranges. (The mirror separations used were $0.4,2,7.5$, and $26 \mathrm{~mm}$.) Raman spectra were collected with a Spex 1401 tandem grating spectrometer equipped with holographic gratings. The exciting light for both experiments was the $488 \mathrm{~nm}$ line of an argon laser (Coherent Innova 90 or Cohernet model 52).

The four sets of interferometric spectra and the Raman spectra were combined to provide wide-frequency-range composite spectra, following essentially the same procedures described in Refs. [3] and [4]. Raw data were smoothed in the MCS data acquisition board before being transferred to the Vax 11/780 computer for analysis. For the interferometric data, the Stokes and anti-Stokes sides of the spectra were averaged, while for the Raman data, only Stokes spectra were recorded. A logarithmic interval averaging was performed for individual spectra, and the spectra for different spectral ranges were then joined by matching the overlapping regions. In this process, the Raman spectra and some of the interferometric spectra were carefully controlled to avoid drifts in instrumental response, and these spectra were used to normalize the intensity.

Figure 1 shows the complete set of composite spectra which span the frequency range of $0.2 \mathrm{GHz}$ to $4 \mathrm{THz}$. The narrow peaks near $12 \mathrm{GHz}$ are the intense LA Brillouin modes, which leak slightly through the imperfect polarizer. These spectra are similar to those found for CKN [3] and salol [4]; similar spectra have also been observed in neutron scattering studies of several glassforming materials. The spectra of Fig. 1 were converted to susceptibility spectra $\chi^{\prime \prime}(\omega)$ through the relation

$$
\chi^{\prime \prime}(\omega)=I(\omega) /[n(\omega, T)+1],
$$

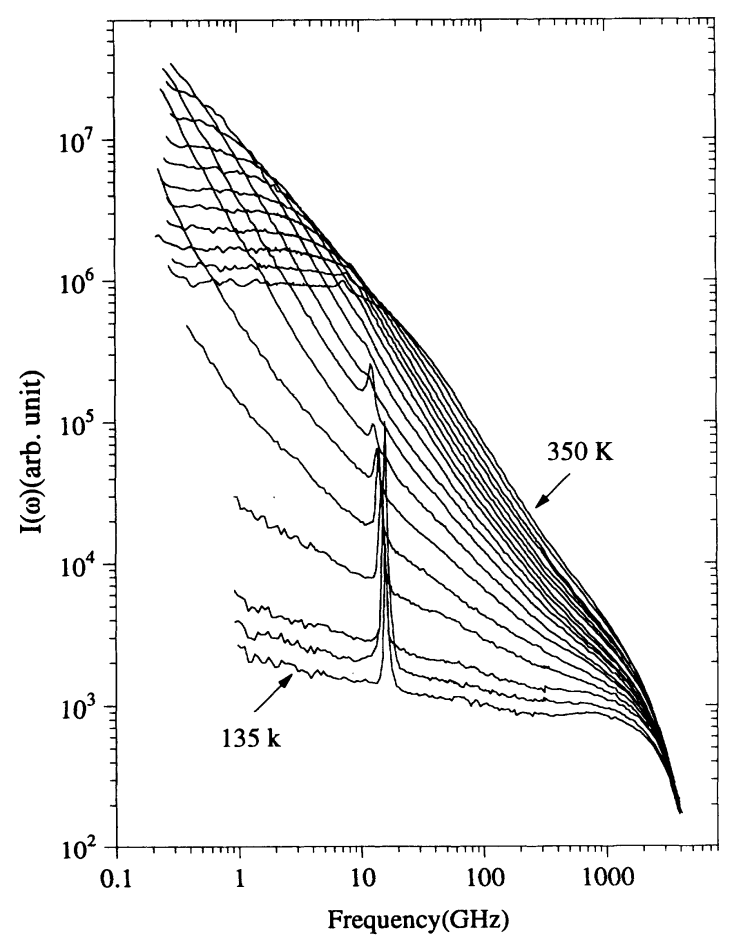

FIG. 1. Composite $I_{\mathrm{VH}}$ spectra of $\mathrm{PC}$ at $\theta=172^{\circ}$. The peaks around $12 \mathrm{GHz}$ are due to leakage of the intense LA Brillouin mode through the imperfect polarizer. The temperatures are (from top to bottom) 350, 330, 310, 295, 280, 270, 260, 250, 240, $230,220,210,200,190,180,170,160,150$, and $135 \mathrm{~K}$. 
where $n(\omega, T)$ is the Bose factor. These $\chi^{\prime \prime}(\omega)$ spectra, shown in Fig. 2, exhibit the evolution of structural relaxation with decreasing temperature identified in other experimental studies and also predicted by MCT. At low frequencies, there is an $\alpha$-relaxation peak associated with the primary structural relaxation process, which moves towards lower frequencies with decreasing temperatures and disappears from our spectral window at $\sim 220 \mathrm{~K}$. The weaker peak at $\sim 1.6 \mathrm{THz}$ is the microscopic band (sometimes called the boson peak). At high temperatures, only the microscopic band and the $\alpha$ peak are visible. With decreasing temperature, as the $\alpha$ peak moves towards lower frequencies, the $\beta$-relaxation process gradually develops in the intermediate-frequency region between the $\alpha$ peak and the microscopic band. At temperatures below $\sim 200 \mathrm{~K}$, the $\alpha$ peak and the minimum of the $\beta$ relaxation have moved out of our spectral window.

Another set of VH spectra was collected with $\theta=90^{\circ}$. These spectra were very similar to the $172^{\circ}$ spectra, except that they were weaker, and the transverse Brillouin components appeared for temperatures below $\sim 190 \mathrm{~K}$. The close agreement between the two sets of spectra demonstrates that the relaxation process responsible for the observed light scattering is $q$ independent in this small-wave-vector limit.

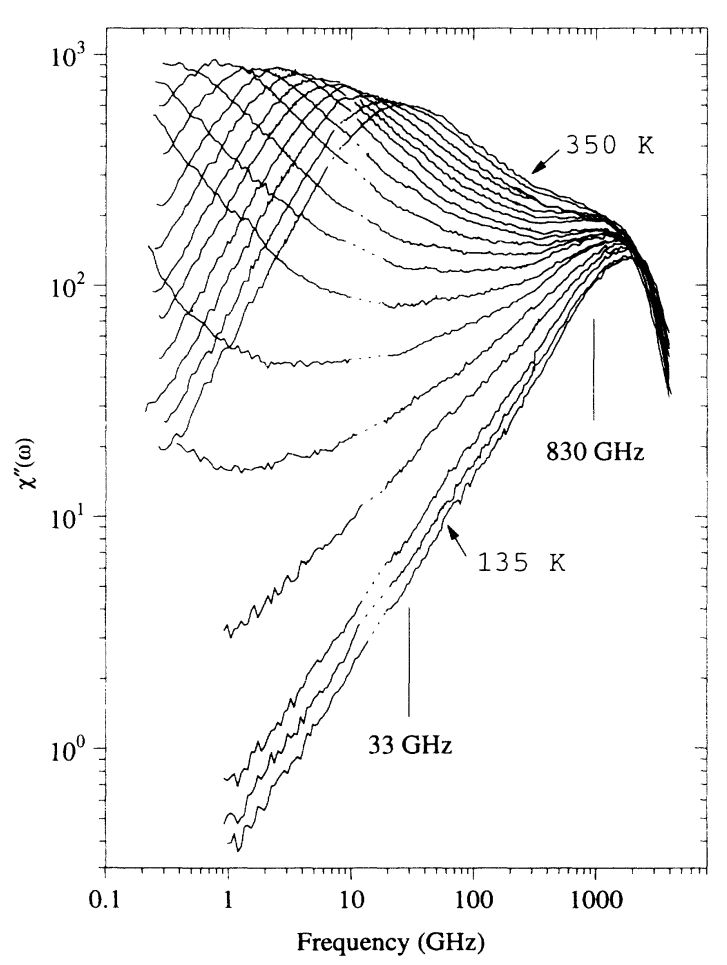

FIG. 2. Susceptibility spectra $\chi^{\prime \prime}(\omega)$ obtained from the data of Fig. 1. The temperatures are the same as in Fig. 1. The broad peaks at low frequencies are the $\alpha$-relaxation peaks. The high-frequency peak at $\sim 1.6 \mathrm{THz}$ is the microscopic excitation band. The $\beta$-relaxation region is located between the $\alpha$ peak and the microscopic band. The lines at 33 and $830 \mathrm{GHz}$ indicate the spectral range selected for the scaling analysis for $T<T_{C}$ as described in the text.

\section{IDEALIZED MODE COUPLING THEORY}

Since extensive reviews of MCT have recently appeared [2,1] and the MCT analysis of depolarized lightscattering spectra has been discussed previously $[3,4]$, we restrict the following discussion to a presentation of those results of the idealized MCT which will be used in the data analysis in Sec. IV. A discussion of the extended MCT will be deferred until Sec. V where it will be combined with the extended MCT data analysis.

The mode coupling theory of the liquid-glass transition, proposed in 1984 [22,23], attributes the ergodic-tononergodic transition to retarded nonlinear interactions among the density fluctuation modes $\rho_{q}(t)$. As the strength of these interactions increases with decreasing temperature, the normalized correlation functions $\phi_{q}(t)=\left\langle\rho_{q}^{*}(t) \rho_{q}(0)\right\rangle /\left\langle\left|\rho_{q}(t)\right|^{2}\right\rangle$ decay towards zero more and more slowly, eventually reaching the glass transition singularity (GTS) at $T=T_{C}$ where the $t \rightarrow \infty$ limit of $\phi_{q}(t)$ increases abruptly from 0 to $f_{q}^{c}$. Below $T_{C}$, it continues to increase as $\left(T_{C}-T\right)^{1 / 2}$.

This $t \rightarrow \infty$ limit, called the nonergodicity parameter or Debye-Waller factor $f_{q}(T)$, determines the elastic fraction of neutron scattering. Since elastic scattering is measured within some small but nonzero energy window, the effective nonergodicity parameter is [2]

$$
f_{q}(T)=f_{q}^{c}+\left\{\begin{array}{l}
h_{q} \sqrt{\sigma}+O(\sigma), \quad T<T_{C}, \\
O(\sigma), \quad T>T_{C},
\end{array}\right.
$$

where $\sigma \propto\left(T_{C}-T\right) / T_{C}$ is the separation parameter which measures the distance from $T_{C}$. The square-root cusp anomaly implied by Eq. (3.1) has been exploited to determine $T_{C}$ in several neutron scattering experiments [2].

The susceptibility spectrum $\chi^{\prime \prime}(\omega)$, which will be of primary concern here, is determined by $\phi_{q}(t)$ through the relation

$$
\chi^{\prime \prime}(\omega)=\omega \operatorname{Re}\left\{\mathscr{F}\left[\phi_{q}(t)\right]\right\},
$$

where $\mathcal{F}$ implies Fourier transformation. For $T>T_{C}$, $\chi^{\prime \prime}(\omega)$ is predicted to display three regions: a highfrequency peak corresponding to microscopic phononlike excitations, a low-frequency primary $\alpha$-relaxation peak which moves towards lower frequencies with decreasing temperature, and between these two an intermediate $\beta$ relaxation region surrounding a minimum. For $T<T_{C}$, the $\alpha$ peak disappears, and the $\beta$-relaxation region changes from concave upwards to concave downwards (a "knee"). This $\beta$-relaxation region, which will be our primary concern, has been shown to possess several crucial properties independent of the details of the microscopic nonlinear interactions.

For $T>T_{C}$, in the region of the minimum, $\chi^{\prime \prime}(\omega)$ can be represented approximately by the interpolation equation

$\chi^{\prime \prime}(\omega)=\chi_{\min }^{\prime \prime}\left[b\left(\omega / \omega_{\min }\right)^{a}+\left(\omega_{\min } / \omega\right)^{b}\right] /(a+b)$.

The two critical exponents $a$ and $b$ in Eq. (3.3) $(0<a<0.4,0<b \leq 1, a<b)$, are both determined by the 
exponent parameter $\lambda$ through

$\lambda=\Gamma^{2}(1-a) / \Gamma(1-2 a)=\Gamma^{2}(1+b) / \Gamma(1+2 b)$.

Although the exponent parameter $\lambda$ can be computed from the structure factors $S(q)$, in practice these are not adequately known for most real materials and $\lambda$ is treated as the one adjustable material-dependent parameter of the idealized MCT.

In the $\beta$-relaxation region $\chi^{\prime \prime}(\omega)$ is predicted to factorize, and also to obey a scaling relation, so that

$$
\chi^{\prime \prime}(\omega)=h_{q}|\sigma|^{1 / 2} \hat{\chi}_{ \pm}^{\prime \prime}\left(\omega / \omega_{\sigma}\right),
$$

where the separation parameter $\sigma \propto\left(T_{C}-T\right)$ and the scaling frequency $\omega_{\sigma} \propto|\sigma|^{1 / 2 a}$.

The two susceptibility master functions $\hat{\chi}_{+}^{\prime \prime}$ for the glass $\left(T<T_{C}\right)$ and $\hat{\chi}_{-}^{\prime \prime}$ for the liquid $\left(T>T_{C}\right)$ can be calculated numerically once $\lambda$ is specified [24]. Equation (3.5) then predicts that for either $T>T_{C}$ or $T<T_{C}$, all susceptibility spectra should scale onto a single master curve, with the scaling frequency $\omega_{\sigma} \rightarrow 0$ as $T \rightarrow T_{C}$ from either above or below.

The results of fits of spectra in the region of the minimum $\left(\omega_{\min }\right)$ for $T>T_{C}$ or in the region of the knee $\left(\omega_{e}\right)$ for $T<T_{C}$ yield the various scaling quantities with predicted dependence on $\sigma$ given by

$$
\begin{aligned}
& \chi_{\min }^{\prime \prime}, \chi_{e}^{\prime \prime} \propto|\sigma|^{1 / 2}=\chi_{\sigma}^{\prime \prime}, \\
& \omega_{\min }, \omega_{e} \propto|\sigma|^{1 / 2 a} / t_{0}=\omega_{\sigma} .
\end{aligned}
$$

In the region of the $\alpha$-relaxation peak, for $T>T_{C}$, MCT predicts another scaling relation

$$
\chi_{q}^{\prime \prime}(\omega)=F_{q}\left(\omega / \omega_{\alpha}\right),
$$

where $\omega_{a}$ is the position of the $\alpha$-relaxation peak. However, there is no factorization in this region. $\omega_{\alpha}$ and the form of $f_{q}$ can depend on both $q$ and the probe, and depend sensitively on the full structure of the memory function.

It was shown by Götze and Sjögren [25] that the stretched-exponential (Kohlrausch) function

$$
\phi_{q}(t) \propto A \exp \left[-\left(t / \tau_{a}\right)^{\beta}\right]
$$

is a good approximate solution to the MCT equations in the $\alpha$-relaxation region. If the stretching constant $\beta$ (which may depend on $q$ ) is temperature independent, then Eq. (3.8) automatically satisfies the scaling prediction Eq. (3.7), with frequency at each temperature scaled by $\omega_{\alpha}=1 / \tau_{a}$ [2]. (This result is also called the timetemperature superposition principle).

Finally, the $\alpha$-relaxation frequency $\omega_{\alpha}$ is predicted to approach 0 as $T \rightarrow T_{C}^{+}$as

$$
\omega_{\alpha} \propto|\sigma|^{\gamma}
$$

with $\gamma=(1 / 2 a)+(1 / 2 b)$.

Note that the equations in this section are asymptotic results derived within the idealized MCT for small frequencies $\omega$ and small separation parameter $\sigma$. Their validity breaks down with increasing $|\sigma|$; it also breaks down for very small $|\sigma|$ and small $\omega$ where the idealized
MCT is no longer a valid approximation to the complete MCT, as we will discuss below.

\section{DATA ANALYSIS WITH IDEALIZED MCT}

\section{A. $\boldsymbol{\beta}$ relaxation}

It is useful to begin the data analysis on the basis of the above results of the idealized MCT, even though they are only an approximation to the complete theory. First, the equations are simple and easy to manipulate. Second, they provide estimates for $T_{C}$ and $\lambda$ which are needed as a starting point for the extended MCT analysis. Of course in this analysis the data for small $|\sigma|$ and $\omega$, which are sensitive to hopping effects, may deviate from the predictions of Sec. III where hopping is neglected.

The susceptibility spectra of Fig. 2 exhibit a susceptibility minimum for temperatures between 190 and $270 \mathrm{~K}$, as expected for the $\beta$-relaxation process when $T>T_{C}$. We first fit the nine spectra in this temperature range using the interpolation equation (3.3). For each spectrum $\omega_{\min }(T)$ and $\chi_{\min }^{\prime \prime}(T)$ were varied independently, but a single optimum value for the critical exponent $a$ was found by fitting all nine spectra simultaneously, with the exponent $b$ constrained via Eq. (3.4). The best fit for $a$ was $a=0.29$, from which $b=0.50$ and $\lambda=0.78$ [24]. These nine spectra, together with the interpolation fits, are shown in Fig. 3. The values of $\omega_{\min }(T)$ and $\chi_{\min }^{\prime \prime}(T)$ found from the fits are listed in Table I. In Fig. 3, the frequency range over which the theory and experiment agree expands with decreasing $T$. The expansion verifies an important result of MCT concerning the approach towards the glass transition singularity. For $190 \mathrm{~K}$ the theory describes the data over a three-decade frequency interval. For frequencies below $\sim 0.5 \mathrm{GHz}$ at low temperatures, one notices that the data tend to lie above the idealized MCT curves. This is what one expects near $T_{C}$ as a result of the neglect of hopping processes [26,5]. In Sec. V we will show that when hopping effects are included the discrepancy can be eliminated.

We next constructed the susceptibility master functions $\chi_{ \pm}^{\prime \prime}\left(\omega / \omega_{\sigma}\right)$ of Eq. (3.5) for $\lambda=0.78$ using the numerical tables of Götze [24]. The nine spectra for $190 \leq T \leq 270 \mathrm{~K}$ were scaled onto the $\chi_{-}^{\prime \prime}\left(\omega / \omega_{\sigma}\right)$ function as shown in Fig. 4(a); the values of $\omega_{\sigma}$ and $\chi_{\sigma}^{\prime \prime}$ found from the scaling fits are listed in Table $I$. To test the accuracy of the exponent parameter $\lambda=0.78$, two other master functions for $\lambda=0.70$ and $\lambda=0.84$ were constructed and are also plotted in Fig. 4(a). The large disagreement seen for these two master functions indicates that the precision for $\lambda$ is $\lambda=0.78 \pm 0.05$, and this implies that the exponents $a=0.29 \pm 0.03, b=0.50 \pm 0.09$.

The scaling analysis for $T<T_{C}$ is much less clear than for $T>T_{C}$ since there is no susceptibility minimum visible within the frequency range of our spectra. Furthermore, hopping effects imply much larger corrections to the idealized MCT results of Eq. (3.5) for $T<T_{C}$ than for $T>T_{C}[26,5]$. To apply the idealized results for $T<T_{C}$, we proceeded as follows. We selected a center frequency of $166 \mathrm{GHz}$ which is one-tenth of the microscopic peak frequency, and performed the scaling analysis on the data 
TABLE I. Fit parameters for the $\beta$ and $\alpha$ relaxation in PC.

\begin{tabular}{|c|c|c|c|c|c|c|c|}
\hline \multirow[b]{2}{*}{$T(\mathbf{K})$} & \multicolumn{4}{|c|}{$\beta$ relaxation } & \multicolumn{3}{|c|}{$\alpha$ relaxation } \\
\hline & $\frac{\omega_{\min }}{2 \pi}(\mathrm{GHz})$ & $\chi_{\min }^{\prime \prime}$ & $\frac{\omega_{\sigma}}{2 \pi}(\mathrm{GHz})$ & $\chi_{\sigma}^{\prime \prime}$ & $\frac{\omega_{\max }}{2 \pi}(\mathrm{GHz})$ & $\tau_{\alpha}(\mathrm{ns})$ & $\beta$ \\
\hline 350 & & & & & 27.5 & $4.85 \times 10^{-3}$ & 0.737 \\
\hline 330 & & & & & 20.7 & $6.45 \times 10^{-3}$ & 0.734 \\
\hline 310 & & & & & 14.8 & $9.31 \times 10^{-3}$ & 0.753 \\
\hline 295 & & & & & 10.3 & $1.35 \times 10^{-2}$ & 0.788 \\
\hline 280 & & & & & 7.19 & $2.00 \times 10^{-2}$ & 0.823 \\
\hline 270 & 728 & 1.92 & 311 & 1.57 & 5.39 & $2.63 \times 10^{-2}$ & 0.825 \\
\hline 260 & 596 & 1.83 & 244 & 1.51 & 3.83 & $3.69 \times 10^{-2}$ & 0.804 \\
\hline 250 & 511 & 1.69 & 227 & 1.38 & 2.68 & $5.36 \times 10^{-2}$ & 0.827 \\
\hline 240 & 381 & 1.63 & 162 & 1.33 & 1.56 & $8.86 \times 10^{-2}$ & 0.789 \\
\hline 230 & 260 & 1.49 & 114 & 1.22 & 0.882 & $1.65 \times 10^{-1}$ & 0.803 \\
\hline 220 & 122 & 1.34 & 51.9 & 1.10 & 0.319 & $4.37 \times 10^{-1}$ & 0.780 \\
\hline 210 & 68.4 & 1.12 & 30.9 & 0.927 & & 1.33 & 0.760 \\
\hline 200 & 29.3 & 0.816 & 12.4 & 0.678 & & & \\
\hline 190 & 5.60 & 0.455 & 2.19 & 0.380 & & & \\
\hline 180 & & & 12.4 & 0.645 & & & \\
\hline 170 & & & 39.4 & 0.844 & & & \\
\hline 160 & & & 105 & 1.04 & & & \\
\hline 150 & & & 151 & 1.14 & & & \\
\hline 135 & & & 208 & 1.30 & & & \\
\hline
\end{tabular}

in the range from $\frac{1}{5}$ to 5 times this frequency $(33-830$ $\mathrm{GHz}$ ) indicated by the two lines in Fig. 2. For PC, as for salol [4], the crossover from $\chi^{\prime \prime}(\omega) \propto \omega$ to $\chi^{\prime \prime}(\omega) \propto \omega^{a}$ predicted by the idealized MCT is completely obscured by hopping effects at low frequencies and by the microscopic structure at high frequencies. It was therefore not possible to carry out a scaling analysis with the $\chi^{\prime \prime}(\omega)$ data alone as we had done for CKN [3]. Since the PC susceptibility spectra in this region are nearly linear on the loglog plot of Fig. 2, we computed the average slope $d \log _{10}\left(\chi^{\prime \prime}\right) / d \log _{10}(\omega)$ in this range, and shifted the center of the data (at $166 \mathrm{GHz}$ ) to the point on the $\chi_{+}^{\prime \prime}(\omega)$ master curve having the same slope. This procedure produced the $\omega_{\sigma}$ and $\chi_{\sigma}^{\prime \prime}$ scaling values for $T<T_{C}$ listed in Table I. The scaling results are shown in Fig. 4(b). Severe discrepancies are evident at low frequencies, as expected for the idealized MCT.

In Fig. 5 we show the frequency of the susceptibility minimum $\omega_{\min }$ for $T>T_{C}$ obtained from the interpolation fits, and the scaling frequency $\omega_{\sigma}$ for both $T>T_{C}$ and $T<T_{C}$ obtained from the scaling fits, each plotted as $\omega^{2 a}$ vs $T$ to test the MCT prediction of Eq. (3.6). Similarly, in Fig. 6, we plot $\chi_{\min }^{\prime \prime 2}$ and $\chi_{\sigma}^{\prime \prime 2}$ vs $T$. Good linear fits are obtained in both figures, all extrapolating to a common zero at $T_{C}=187 \pm 5 \mathrm{~K}$.

\section{B. $\alpha$ relaxation}

In the susceptibility spectra of Fig. 2, an $\alpha$-relaxation peak is clearly visible for temperatures above $220 \mathrm{~K}$. $\alpha$ peaks are frequently fit to stretched-exponential functions [Eq. (3.8)] with $A, \tau_{\alpha}$, and $\beta$ treated as fitting parameters. MCT has shown that Eq. (3.8), while not an exact solution of the MCT equations, provides a quite accurate representation of the $\alpha$-relaxation spectrum. The scaling prediction of MCT [Eq. (3.7)] is automatically satisfied by Eq. (3.8) if $\beta$ is independent of temperature.

In Fig. 7 we show the susceptibility spectra of Fig. 2 for temperatures between 210 and $350 \mathrm{~K}$ together with the fits to Eqs. (3.2) and (3.8). The fit results for $\beta, \tau_{\alpha}$, and $\omega_{\max }$ at each temperature are listed in Table I. Note that the high-frequency wings of the $\alpha$ peaks fall systematically above the fits, due to the onset of the $\beta$ relaxation process. (Also note that in this part of the spectrum, the $\alpha$-relaxation and $\beta$-relaxation regions overlap.)

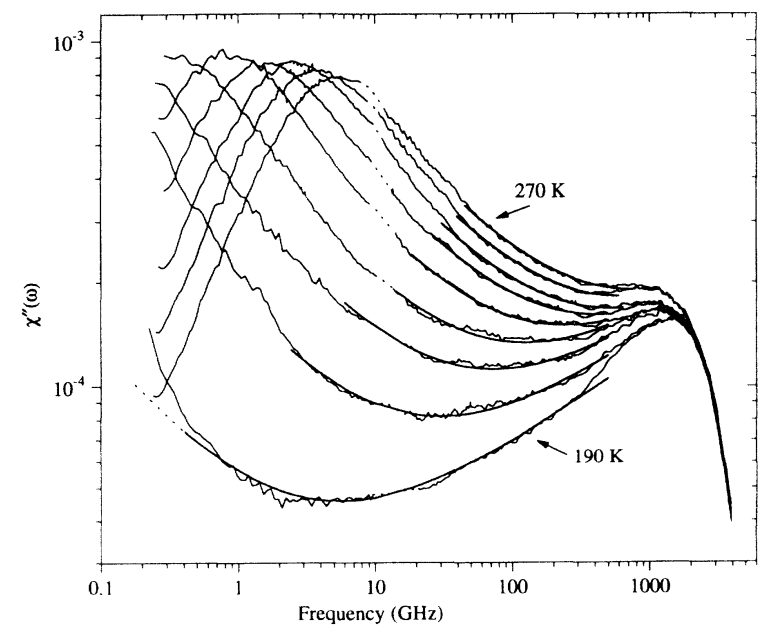

FIG. 3. Experimental susceptibility spectra for $190 \leq T \leq 270$ K fitted by the idealized MCT interpolation equation (3.3) in the $\beta$-relaxation region with $a=0.29$ and $b=0.50 . \quad(\lambda=0.78$. ) For the $190 \mathrm{~K}$ curve, the MCT fit has been extended beyond the fitting region (dotted line) to illustrate the disagreement resulting from the neglect of hopping effects. 

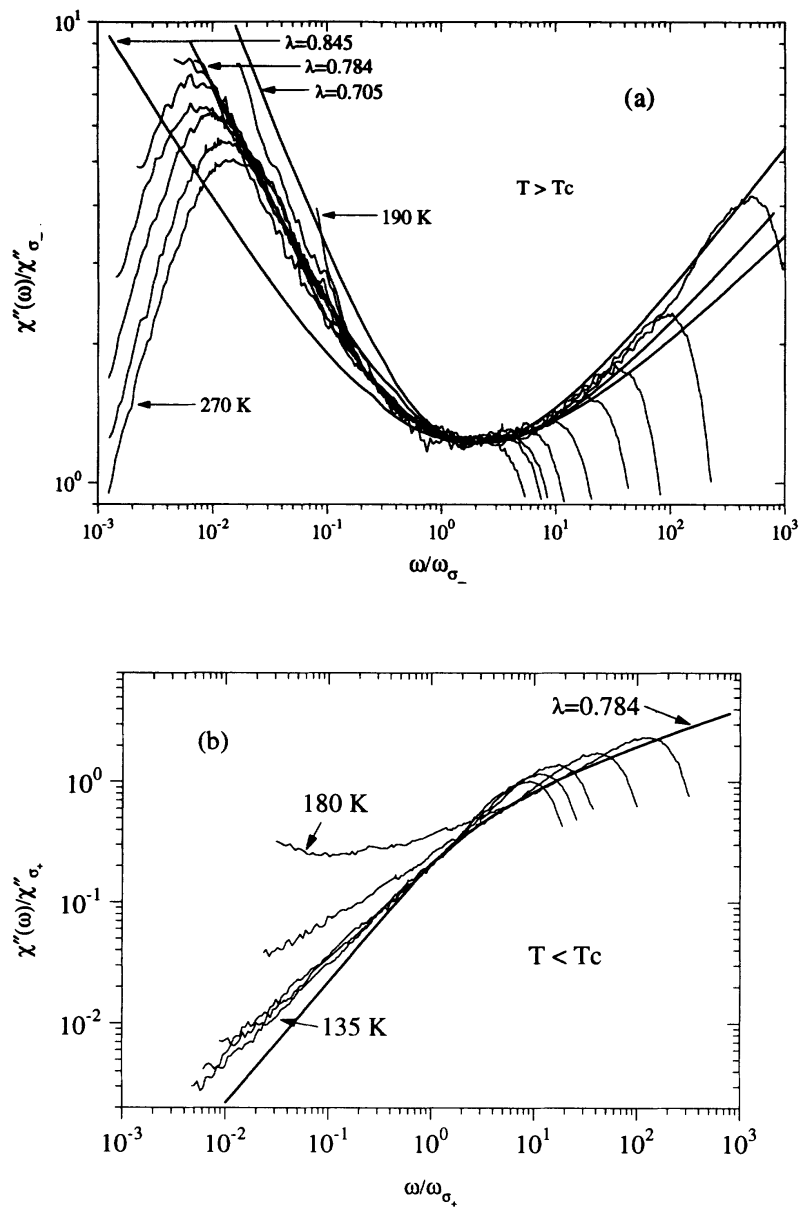

FIG. 4. Susceptibility spectra shifted to overlap at the minima, together with the MCT master functions $\chi_{ \pm}^{\prime \prime}\left(\omega / \omega_{\sigma}\right)$ for $\lambda=0.78$. (a) $\left(T>T_{C}\right)$ : temperatures are (from top to bottom), 190 to $270 \mathrm{~K}$ in steps of $10 \mathrm{~K}$. To demonstrate the accuracy of the exponent parameter $\lambda$, two other master functions with $\lambda=0.70$ and 0.84 are also plotted. (b) $\left(T<T_{C}\right)$ : Scaling of the portion of the $\chi^{\prime \prime}(\omega)$ spectra between the vertical lines in Fig. 2 onto a $\lambda=0.78$ master function. Temperatures are (from top to bottom) $180,170,160,150$, and $135 \mathrm{~K}$.

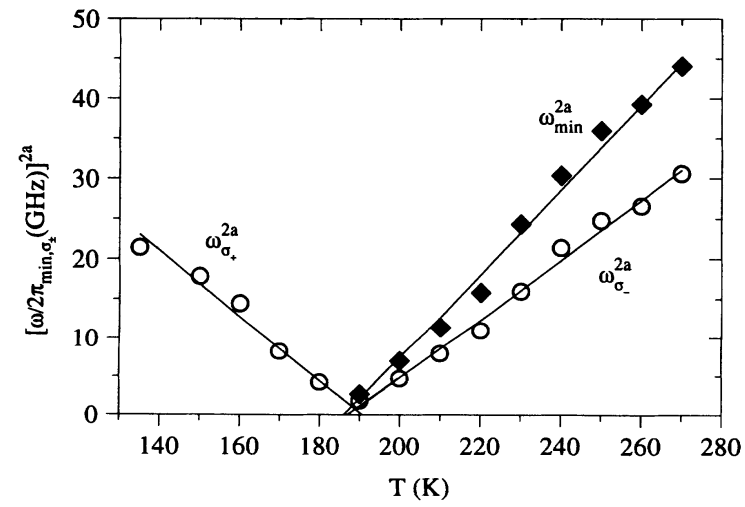

FIG. 5. $\omega_{\min }^{2 a}$ vs $T\left(\vartheta, T>T_{C}\right)$ and $\omega_{\sigma}^{2 a}$ vs $T\left(0, T \gtrless T_{C}\right)$. Extrapolation to $\omega=0$ gives $T_{C}=187 \pm 5 \mathrm{~K}$.

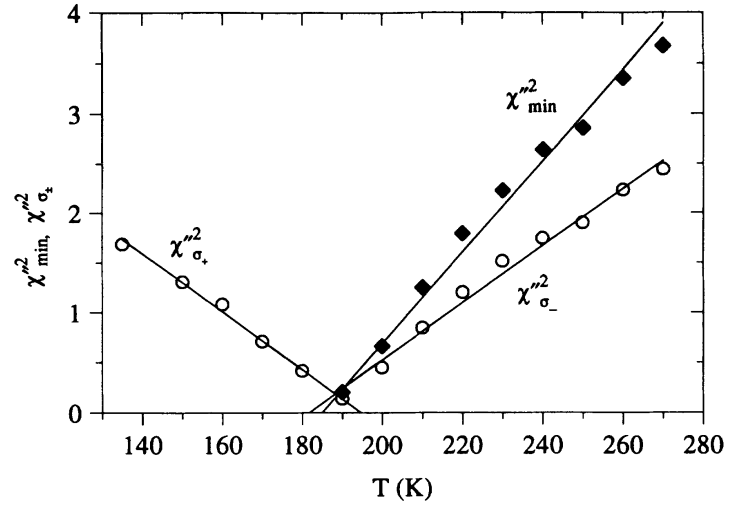

FIG. 6. $\chi_{\min }^{\prime \prime 2}$ vs $T\left(\diamond, T>T_{C}\right)$ and $\chi_{\sigma}^{\prime \prime 2}$ vs $T\left(0, T \gtrless T_{C}\right)$. Extrapolation to $\chi^{\prime \prime}=0$ gives $T_{C}=187 \pm 5 \mathrm{~K}$.

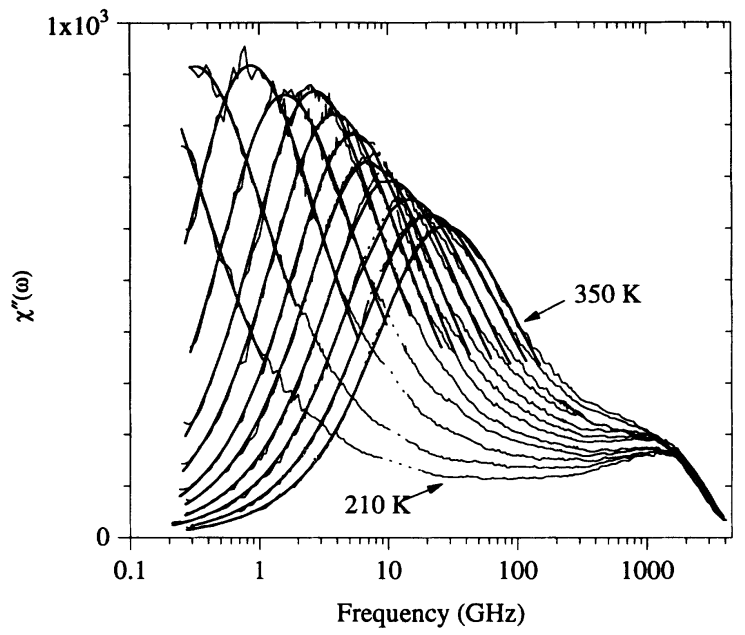

FIG. 7. $\alpha$-relaxation region of the $\chi^{\prime \prime}(\omega)$ spectra of Fig. 2 fitted with Eqs. (3.2) and (3.8) for temperatures (from right to left) $350,330,310,295,280,270,260,250,240,230,220$, and $210 \mathrm{~K}$.

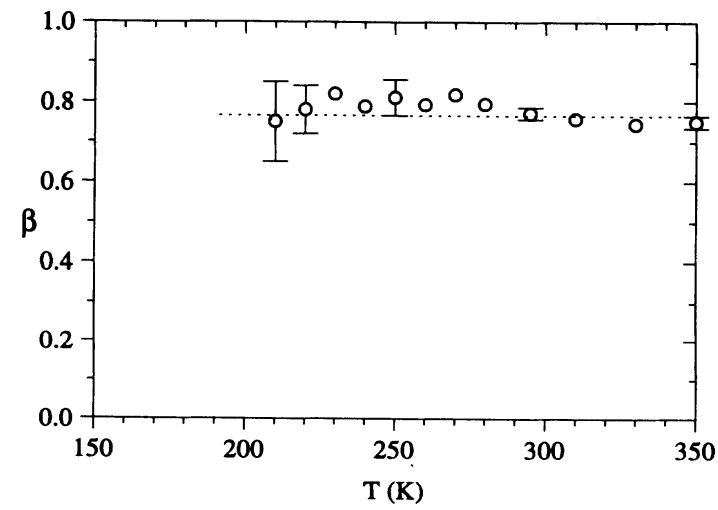

FIG. 8. Stretching constant $\beta$ from the fits in Fig. 7. The constant $\beta$ fit indicated by the dashed line gave $\beta=0.77 \pm 0.05$. 
The stretching constant $\beta$ found from these fits is plotted against temperature in Fig. 8. The data indicate that $\beta=0.77 \pm 0.05$ with no evidence of a systematic increase with increasing temperature. Borjesson, Elmroth, and Torell [8] also found a temperature-independent $\beta$ from their Brillouin scattering study of PC, although with a somewhat smaller value $(\beta \sim 0.55)$. (Note that MCT predicts that $\beta$ can be different for different quantities.) These results obey the scaling (i.e., time-temperature superposition) of $\alpha$ relaxation predicted by MCT.

The MCT prediction for the temperature dependence of $\omega_{\max } \approx \omega_{\alpha}=1 / \tau_{\alpha}$ is given by Eq. (3.9). The values of $a$ and $b$ found from the analysis of the $\beta$-relaxation region in the preceding section fix $\gamma=1 / 2 a+1 / 2 b=2.74$. In Fig. 9 we plot $\tau_{\alpha}^{-1 / 2.74}$ vs $T$. The data show the predicted linear temperature dependence, yielding $T_{C}=186 \pm 3 \mathrm{~K}$, which agrees, within experimental error, with the result obtained from the analysis of the $\beta$-relaxation region.

We have also converted viscosity data [19] to a viscous relaxation time $\tau_{\eta}$ with the Stokes-Einstein relation: $\tau_{\eta}=4 \pi R^{3} \eta / 3 k_{B} T$, fixing $R=1.7 \AA$ to match $\tau_{\alpha}$ at high temperatures. The high-temperature data, again plotted

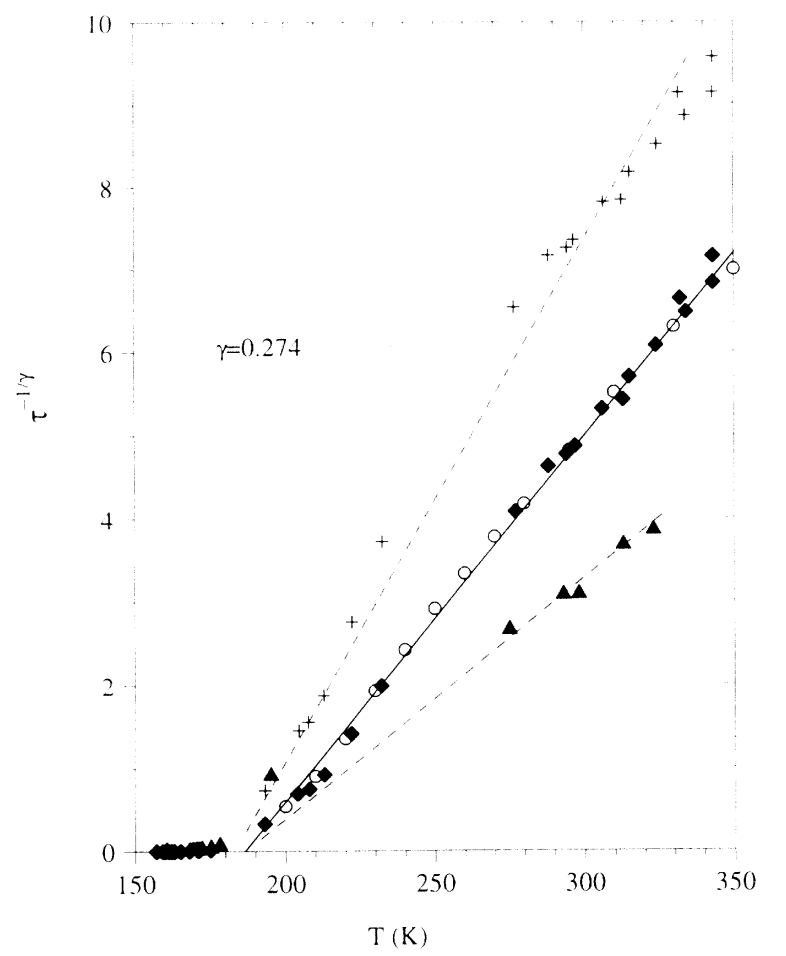

FIG. 9. $\alpha$-relaxation time $\tau_{\alpha}$ obtained from the fits in Fig. 7 plotted as $\tau_{\alpha}^{-1 / \gamma}$ with $\gamma=2.74$ vs $T(O)$. The best straight line fit gave $T_{C}=186 \mathrm{~K}$. Viscosity data $(\diamond)$ [19] was converted to relaxation time $\tau_{\eta}$ with the Stokes-Einstein relation $\tau_{\eta}=4 \pi R^{3} \eta /\left(3 k_{B} T\right)$ where $R=1.7 \AA$ was obtained by matching our $\alpha$-relaxation time $\tau_{\alpha}$ with $\tau_{\eta}$ at high temperatures. A linear fit gave $T_{C}=188 \pm 3 \mathrm{~K}$. (+) are scaled $\tau$ values calculated from the same viscosity data [19], but using the Maxwell relation $\tau=\eta / G_{\infty} . G_{\infty}$ was obtained from $G_{\infty}=\rho V_{\mathrm{TA}}^{2}$ where $V_{\mathrm{TA}}$ was determined from $90^{\circ}$ depolarized Brillouin spectra (temperature from 125 to $190 \mathrm{~K}$ ) and extrapolated to high temperatures by assuming a linear temperature dependence. $(\boldsymbol{\Delta})$ are scaled $\tau$ values from dielectric data $[14,12,13,16]$. All three plots give a critical temperature around $185 \mathrm{~K}$. as $\tau_{\eta}^{-1 / 2.74}$ vs $T$, are approximately linear, and extrapolate to zero at $T_{C}=188 \pm 3 \mathrm{~K}$, close to the $\tau_{\alpha}$ result. This fit is also shown in Fig. 9 along with a fit of dielectric data.

\section{EXTENDED MCT ANALYSIS}

The idealized MCT discussed in Sec. III is a convenient but incomplete approximation to the full MCT. The idealized theory contains only nonlinear interactions among density fluctuation modes in the memory kernel, representing the cage effect, and predicts a complete structural arrest at $T_{C}$. Following a suggestion by Das and Mazenko [27], Götze and Sjögren [28] identified the leading correction terms to the memory kernel, and found a set of closed equations of motion for the $\Phi_{q}(t)$ which constitute the extended MCT. These terms, which represent ergodicity-restoring activated transport processes, appear in the resulting equation of motion for the $\beta$ correlator $G(t)$ as a temperature-dependent hopping parameter $\delta(T)$. In the extended theory the sudden structural arrest at $T_{C}$ is replaced by a gradual crossover from liquid dynamics to glassy dynamics; the $\alpha$ peak and susceptibility minimum both exist at all temperatures. The structure and scaling properties of the extended MCT $\beta$ correlator are discussed in detail in Ref. [29]. The extended theory reduces to the idealized version in the limit $\delta \rightarrow 0$.

In the extended MCT, the one-parameter scaling law for $\chi^{\prime \prime}(\omega, \sigma)$ of Eq. (3.5) is replaced by a two-parameter scaling law for $\chi^{\prime \prime}\left(\omega, \sigma, \delta t_{0}\right)$ where $t_{0}$ is a microscopic matching time that makes the hopping parameter $\delta t_{0}$ dimensionless. (The choice of $t_{0}$ has no effect on the form of $\chi^{\prime \prime}$ which is completely determined by $\lambda$.)

In carrying out fits to experimental susceptibility data with the extended MCT, the exponent parameter $\lambda$ is fixed, and the three parameters $h(T), \sigma(T)$, and $\delta t_{0}(T)$ are varied to optimize the fit at each temperature to

$$
\chi^{\prime \prime}(\omega)=h_{q} \hat{\chi}^{\prime \prime}\left(\omega, \sigma, \delta t_{0}\right) .
$$

In Ref. [5] extended MCT fits were carried out for CKN and salol, and it was found that in the vicinity of $T_{C}$ both $h(T)$ and $\sigma(T)$ could be represented by linear functions of $T$, while $\delta t_{0}(T)$ was approximated by an Arrhenius function. It was also found that the optimum exponent parameters were slightly larger than for the idealized theory: $\lambda=0.85$ (rather than 0.81 ) for CKN, and $\lambda=0.73$ (rather than 0.70 ) for salol.

This fitting procedure also established the trajectories in the $\left(\sigma, \delta t_{0}\right)$ parameter space followed by each of these systems as $T$ changes. These trajectories cut across different scaling lines where $\delta t_{0}=C|\sigma|^{(1+2 a) / 2 a}$ as $T$ decreases so that simple $T$ scaling does not fully apply, which explains why the idealized MCT scaling is only approximate.

An extended MCT analysis was carried out for PC following the procedures described in Ref. [5] for CKN and salol. Fits were performed with two values of $\lambda, \lambda=0.78$ (found in the idealized MCT analysis) and $\lambda=0.81$. With $\lambda=0.81$ it was not possible to obtain good fits to the data for $T$ well above $T_{C}$ with $\delta=0$. We therefore completed 
the fitting procedure with $\lambda=0.78$. The fits are shown in Fig. 10. Note that inclusion of $\delta \neq 0$ has eliminated disagreements of the idealized MCT fits found in Figs. 3 and 4. The MCT result of Eq. (5.1) accounts for the $\beta$ spectrum for $T<T_{C}$ down to the lowest frequencies we measured. The values of $\sigma$ and $\delta t_{0}$ from these fits are given in Table II.

As discussed in Ref [5], the supercooled liquid dynamics pass smoothly from the high-temperature liquid region to an intermediate crossover region where the cage and hopping effects are comparable, and then from the intermediate region to the low-temperature glassy region where the hopping effects dominate. The intermediate crossover region occurs between $\sigma=-\sigma_{0}$ and $\sigma=+\sigma_{0}$ where the hopping-induced scale $\sigma_{0}$ is set by

$$
\sigma_{0}=\left(\delta t_{0}\right)^{(2 a) /(1+2 a)} \text {. }
$$

In Fig. 11(a) we show the separation parameter $\sigma(T)$ found from the fits, together with the $\pm \sigma_{0}$ lines determined from the $\left(\delta t_{0}\right)$ values via Eq. (5.2). The zero of $\sigma(T)$ defines the critical temperature and we find $T_{C}=179 \pm 2 \mathrm{~K}$. This plot locates the crossover region centered at $T_{C} \sim 179 \mathrm{~K}$ with a width of $\sim 10 \mathrm{~K}$, comparable to the salol results in Ref. [5]. The transition regions for $\mathrm{PC}$ and salol are much smaller than the $\mathrm{CKN}$ result of $\sim 70 \mathrm{~K}$. The amplitude $h(T)$ is shown in Fig. 11(b). Although the temperature dependence in the vicinity of $T_{C}$ is steep, it is still well represented by a linear function of $T$ as found for CKN and salol.

In Fig. 12(a) we show the $\left(\sigma, \delta t_{0}\right)$ values found from

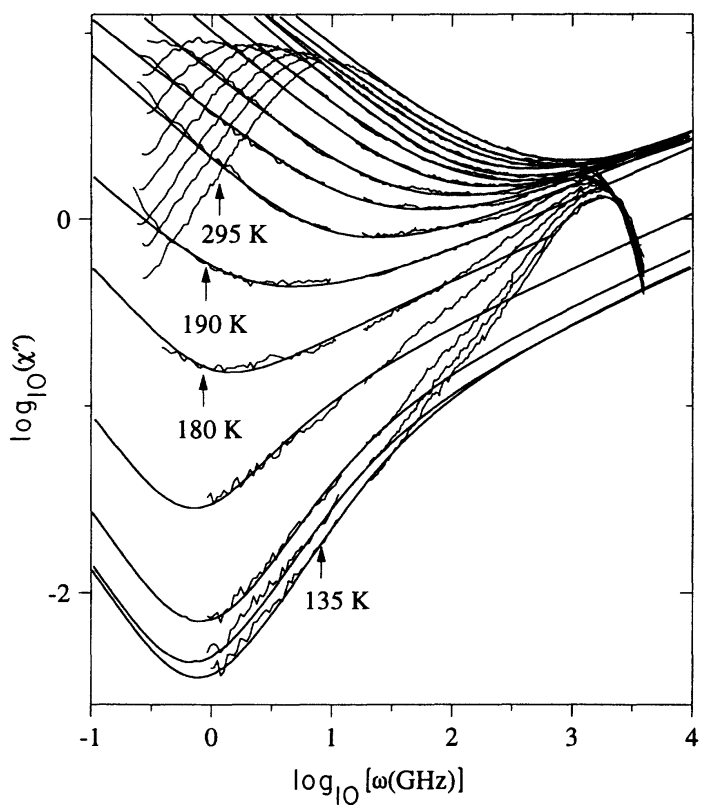

FIG. 10. Susceptibility spectra $\chi^{\prime \prime}(\omega)$ of PC fitted by $\delta \neq 0 \beta$ relaxation functions of the extended MCT. The fits use the same exponent $a=0.29$ corresponding to $\lambda=0.78$ obtained in the interpolation fits of Fig. 3. The temperatures are (from top to bottom) $295,280,270,260,250,240,230,220,210,200,190$, $180,170,160,150$, and $135 \mathrm{~K}$. The arrows at 180 and $190 \mathrm{~K}$ indicate the increasing effects of hopping processes in the crossover region.
TABLE II. Fitting parameters for extended MCT analysis $\left[t_{0}=(1 / 2 \pi) \mathrm{ps}\right]$.

\begin{tabular}{cccl}
\hline \hline$T(\mathbf{K})$ & $\sigma$ & $\delta t_{0}$ & $h$ \\
\hline 135 & 0.0904 & $7.16 \times 10^{-7}$ & 0.527 \\
150 & 0.0692 & $6.92 \times 10^{-7}$ & 0.513 \\
160 & 0.0622 & $1.03 \times 10^{-6}$ & 0.636 \\
170 & 0.0238 & $1.51 \times 10^{-6}$ & 0.982 \\
180 & 0.0039 & $5.55 \times 10^{-6}$ & 1.64 \\
190 & -0.0247 & $7.56 \times 10^{-6}$ & 2.20 \\
200 & -0.0618 & $9.14 \times 10^{-5}$ & 2.56 \\
210 & -0.126 & $1.78 \times 10^{-5}$ & 2.60 \\
220 & -0.176 & $1.24 \times 10^{-4}$ & 2.61 \\
230 & -0.253 & $7.07 \times 10^{-4}$ & 2.43 \\
240 & -0.353 & $5.86 \times 10^{-4}$ & 2.22 \\
250 & -0.339 & $4.73 \times 10^{-3}$ & 2.36 \\
260 & -0.367 & $5.23 \times 10^{-3}$ & 2.48 \\
270 & -0.527 & & 2.16 \\
280 & -0.571 & & 2.15 \\
295 & -0.652 & & 2.10 \\
\hline \hline
\end{tabular}
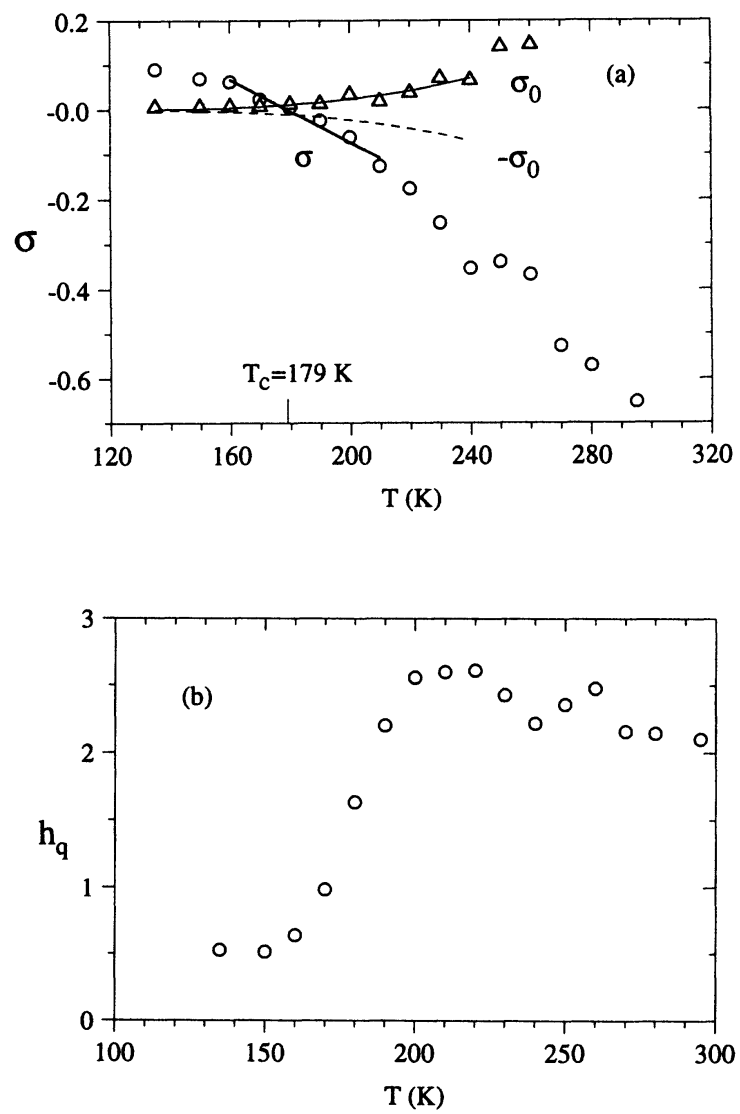

FIG. 11. Parameters from extended MCT fits. (a) Separation parameter $\sigma(0)$ and the hopping-induced scale $\pm \sigma_{0}(\Delta)$. The heavy solid line is a linear fit $\sigma=A\left(T_{C}-T\right)$ in the temperature range $160 \leq T \leq 210 \mathrm{~K}$ with $T_{C}=179 \mathrm{~K}$. The solid (broken) line is an Arrhenius fit to $\sigma_{0}\left(-\sigma_{0}\right)$ in the same temperature range. $T=175$ and $185 \mathrm{~K}$ are estimated boundaries of the transition region. (b) The critical amplitude $h_{q}(T)$ vs $T$ from the extended MCT fits. 
the fits (circles) together with an interpolated trajectory found from a linear fit to $\sigma(T)$ and an Arrhenius fit to $\delta t_{0}(T)$. In the figure we also show a series of scaling lines $\delta t_{0} \propto|\sigma|^{(1+2 a) / 2 a}$ for $\sigma / \sigma_{0}= \pm 7.0, \pm 1.87, \pm 1.0$, and \pm 0.33 with increasing steepness. The intersections of the system trajectory $C$ with the $\sigma / \sigma_{0}= \pm 1.0$ scaling line (solid) delineates the crossover region.

The idealized MCT prediction of Eq. (3.5), with $h_{q}$ assumed constant, leads to the prediction that $a_{\text {eff }}$, the slope of $\log _{10}\left(\chi_{\min }^{\prime \prime}\right)$ vs $\log _{10}\left(\omega_{\min }\right)$ should be equal to $a$. However, since $h(T)$ is actually an increasing function of $T, \chi_{\min }^{\prime \prime}$ will increase faster than $|\sigma|^{1 / 2}$ and $a_{\text {eff }}$ should be larger than $a$. This result $a_{\text {eff }}>a$ was found in our previous studies of CKN [3] and salol [4], and was explained on the basis of the temperature dependence of $h(T)$ in Ref. [5].
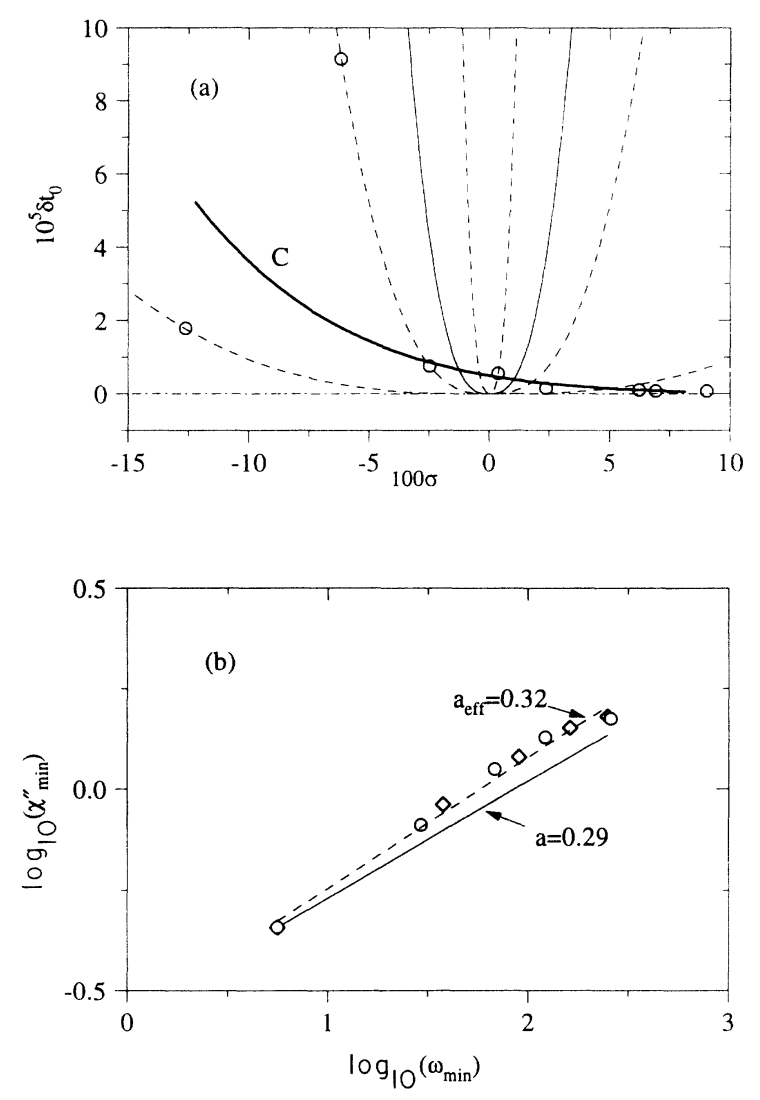

FIG. 12. (a) Trajectory of PC in the $\left(\sigma, \delta t_{0}\right)$ control parameter space in the temperature range $130 \leq T \leq 210 \mathrm{~K}$. The fit results are indicated by $(O)$. The bold line shows the interpolated path $C$ constructed from a linear fit of $\sigma(T)$ and an Arrhenius fit of $\delta t_{0}(T)$. The four generalized parabolas are scaling lines with $\sigma / \sigma_{0}=7.01,1.88,1.00$, and 0.33 (with increasing steepness). The horizontal dot-dashed line indicates the idealized liquid (left) and glass (right) of the $\delta=0$ theory. (b) Double logarithmic plot of $\chi_{\min }^{\prime \prime}$ vs $\omega_{\min }$ with the experimental values shown by (O). A linear fit shown by the broken line gave $a_{\text {eff }}=0.32$, in clear disagreement with the idealized MCT prediction $a_{\text {eff }}=a=0.29$ shown by the solid line. The symbols $(\diamond)$ indicate the modification of the prediction when the temperature dependence of $h(T)$, shown in Fig. 11(b), is included as discussed in the text.
In Fig. 12(b) we show the experimental $\left[\log _{10}\left(\chi_{\min }^{\prime \prime}\right), \log _{10}\left(\omega_{\min }\right)\right]$ values for PC by open circles, with a linear fit which gave $a_{\text {eff }}=0.32$. A straight line with slope $a=0.29$, the value found in Sec. IV, passing through the lowest point is also shown to illustrate that $a_{\text {eff }}>a$.

We recalculated the predictions of Eq. (3.5) with $a=0.29$ including the temperature dependence of $h(T)$ shown in Fig. 11(b). The results are shown by the $(\diamond)$ symbols in Fig. 12(b). Thus the apparent failure of Eq. (3.5) is eliminated when the $T$ dependence of $h(T)$ found in the extended MCT analysis is included.

\section{POLARIZED BRILLOUIN SCATTERING AND ULTRASONICS}

We performed a polarized Brillouin scattering study of PC to explore further the surprising results reported by Elmroth, Borjesson, and Torell [10]. Fuchs, Götze, and Latz [30] had noted that the nonergodicity parameter $f_{q}(T)$ of Eq. (3.1) can be determined in the $q \rightarrow 0$ limit by

$$
f_{0}(T)=1-\left(C_{0} / C_{\infty}\right)^{2},
$$

where $C_{0}$ and $C_{\infty}$ are the sound velocities measured below or above the $\alpha$-relaxation region, respectively. Since MCT predicts that $f_{q}(T)$ should exhibit a squareroot cusp at $T=T_{C}$ for any $q$ [Eq. (3.1)], the temperature-dependent values of $C_{0}$ and $C_{\infty}$ determined from ultrasonic or Brillouin scattering experiments provide an independent method for determining $T_{C}$ in the $q \rightarrow 0$ limit. Three experiments exploiting this approach have been previously reported: (1) Elmroth, Borjesson, and Torell [10] for PC, (2) Dreyfus et al. [31] for salol, and (3) Li et al. [32] for CKN.

In the PC Brillouin scattering experiments of Elmroth, Borjesson, and Torell [10], three aspects of the experimental and data analysis procedures may have contributed to their high value of $T_{C}$. First, the $I_{\mathrm{vv}}$ spectra were analyzed directly without subtraction to remove anisotropic scattering contributions; second, $C_{0}$ and $C_{\infty}$ were both treated as free fitting parameters; and third, structural relaxation was represented by an $\alpha$-relaxationonly model (Cole-Davidson) so that $\beta$-relaxation effects were ignored. In the salol experiment of Dreyfus et al. [31], $C_{0}$ was determined independently from ultrasonic data and the anisotropic contributions were subtracted, producing reasonable agreement between $T_{C}$ found from Eq. (6.1) and from the depolarized light scattering experiment of $\mathrm{Li}$ et al. [4]. In the CKN experiment of $\mathrm{Li}$ et al. [32], we also constructed a memory function which includes the full relaxation dynamics. With this memory function we confirmed Eq. (3.1) with a $T_{C}$ which was consistent with other determinations.

We recorded $\theta=172^{\circ} \mathrm{VV}$ and $\mathrm{VH}$ tandem interferometric spectra of PC with a mirror separation of 6 $\mathrm{mm}(\mathrm{FSR}=25 \mathrm{GHz})$. The $I_{\mathrm{vv}}$ spectra include contributions from longitudinal density fluctuations $I_{\mathrm{LA}}$, anisotropic scattering from orientational fluctuations, and second-order DID scattering [3], while the $I_{\mathrm{VH}}$ spectra contain the orientational and second-order anisotropic 
scattering only. The pure $I_{\mathrm{LA}}$ spectra can be obtained by subtraction:

$$
I_{\mathrm{LA}}=I_{\mathrm{Vv}}-K I_{\mathrm{VH}},
$$

where $K$ is a constant determined from the depolarization ratio at frequencies well above the Brillouin peak [32]. For PC, we found $K=1.24$.

Figure 13 shows the $I_{\mathrm{VV}}$ and $I_{\mathrm{VH}}$ spectra for $T=250$ $\mathrm{K}$, and the corrected $I_{\mathrm{LA}}$ spectrum found with Eq. (6.2). Note that the $I_{\mathrm{LA}}$ spectrum is qualitatively different from the uncorrected $I_{\mathrm{Vv}}$ spectrum at frequencies below the Brillouin peak, indicating that orientational and secondorder scattering processes make significant contributions to $I_{\mathrm{Vv}}$.

The spectrum $I_{\mathrm{LA}}$ of longitudinal density fluctuations including the interaction of LA modes with structural relaxation was analyzed by Mountain [33]. An approximate vẹrsion of Mountain's generalized hydrodynamics result, valid in the spectral region of interest here, is given by $[34,32]$

$$
\begin{aligned}
& I(\omega)=I_{0}\left[\gamma_{0}+m^{\prime \prime}(\omega)\right] / {\left[\omega^{2}-\omega_{0}^{2}+\omega m^{\prime}(\omega)\right]^{2} } \\
&\left.+\left[\omega\left\{\gamma_{0}+m^{\prime \prime}(\omega)\right\}\right]^{2}\right),
\end{aligned}
$$

where $\omega_{0}=C_{0} q$, and $\gamma_{0}$ is a regular damping constant.

The dynamics of structural relaxation enters Eq. (6.3) via the complex memory function $m(\omega)$ which Mountain [33] assumed to be Lorentzian. Frequently, $m(\omega)$ is modeled by a Kohlrausch function or by the closely related Cole-Davidson (CD) function

$$
\omega m(\omega)=\Delta^{2}\left[\left(1-i \omega \tau_{\mathrm{CD}}\right)^{\left.-\beta_{\mathrm{CD}}-1\right]}\right.
$$

where $\Delta^{2}, \tau_{\mathrm{CD}}$, and $\beta_{\mathrm{CD}}$ are treated as adjustable fitting parameters.

In order to reduce the number of free parameters in Eqs. (6.3) and (6.4), we fixed $\beta_{\mathrm{CD}}, \gamma_{0}$, and $C_{0}(T)$ leaving $I_{0}, \Delta^{2}$, and $\tau_{\mathrm{CD}}$ as the three free fitting parameters. From our fits to $\alpha$-relaxation peaks discussed in Sec. IV, we found $\beta=0.77$, which is equivalent [35] to $\beta_{\mathrm{CD}}=0.68$. From the width of the LA Brillouin peak at $130 \mathrm{~K}$ (the lowest temperature studied) we determined $\gamma_{0} / 2 \pi=0.1$ GHz. We assumed that $\boldsymbol{\beta}_{\mathrm{CD}}$ and $\gamma_{0}$ are independent of

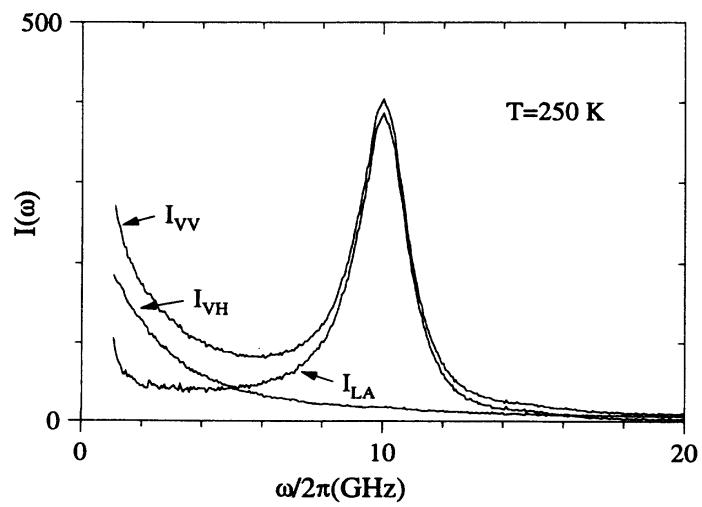

FIG. 13. $\theta=172^{\circ} I_{\mathrm{Vv}}$ and $I_{\mathrm{VH}}$ Brillouin spectra for $T=250$ $\mathrm{K}$ and the corrected longitudinal spectrum $I_{\mathrm{LA}}$ found with Eq. (6.2). Note that the subtraction strongly modifies the shape of the spectrum for frequencies below the Brillouin peak. temperature in this CD model fitting.

To determine independently the low-frequency sound velocity $C_{0}(T)$, ultrasonic measurements were performed at $5 \mathrm{MHz}$ for temperatures between 293 and $240 \mathrm{~K}$. Relative velocities were determined by means of a phasesensitive technique with a precision of $0.01 \%$ [36]. The precision of the absolute velocity was $\sim 1 \%$ due to slight convection of the liquid. Ultrasonic measurements at 5 and $15 \mathrm{MHz}$ down to $213 \mathrm{~K}$ showed measurable dispersion beginning at $\sim 230 \mathrm{~K}$, indicating that measurements at $T \leq 230 \mathrm{~K}$ were no longer determining $C_{0}$ due to the rapidly increasing relaxation time. We therefore fit the measured $C_{0}$ values in the range 293-240 K, where no dispersion was observed, with a straight line, which gave

$$
C_{0}(T)=2507.5-3.612 T(\mathrm{~m} / \mathrm{sec}),
$$

shown by the dashed line in Fig. 14. (Our $C_{0}$ result differs by about $1 \%$ from that reported by Masood et al. [11].)

We performed a conventional nonlinear least-squares fit of the $I_{\mathrm{LA}}$ spectra at each temperature to Eq. (6.3), with the Cole-Davidson approximation of Eq. (6.4) for $m(\omega)$. The ultrasonic $C_{0}(T)$ in Eq. (6.5) was used in the fitting, but with a slight correction by $+0.85 \%$, which is within the experimental error of the ultrasonic measurement, in order to obtain agreement between $\omega_{B}$ and $C_{0} q$ at high temperatures. Fits for five temperatures are shown in Fig. 15. The resulting values of $\tau_{\mathrm{CD}}, \Delta^{2}$, and $C_{\infty}=\left[C_{0}^{2}+(\Delta / q)^{2}\right]^{1 / 2}$ are listed in Table III. The $C_{\infty}$ values are also plotted in Fig. 14 along with $C_{0}$ and with $C_{B}$ determined from the positions of the Brillouin peaks. The nonergodicity parameter $f_{0}(T)_{\mathrm{CD}}$ computed with Eq. (6.1) is shown in Fig. 16 by $(\diamond)$. Note that the fits shown in Fig. 15 are quite good, despite the fact that the fit parameters and $f_{0}(T)$ values are rather different from those in Ref. [10]. This demonstrates the extreme difficulty of inferring the memory function from conventional Brillouin scattering spectra.

As previously noted, the Cole-Davidson approximation, or any model for $m(\omega)$ which neglects $\beta$-relaxation

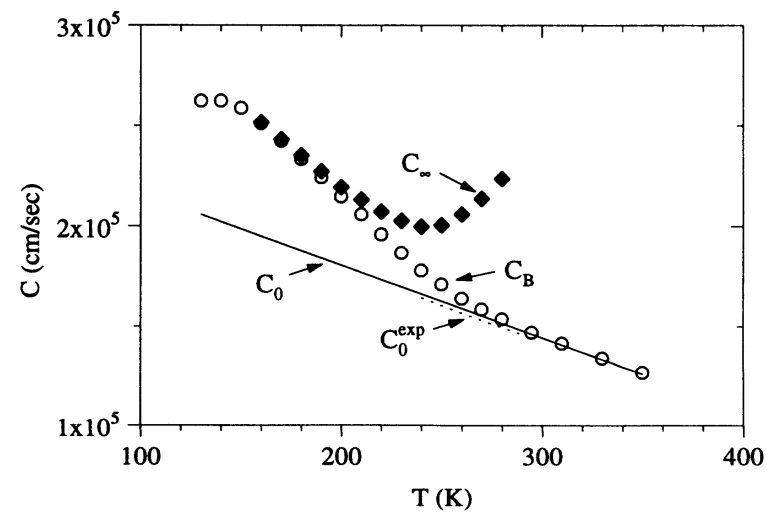

FIG. 14. Sound velocity $C_{0}$ from ultrasonic measurements (dotted line), $C_{B}$ from Brillouin peak positions (O), $C_{\infty}$ from the fits of Fig. $15(\nabla)$. The solid line is the adjusted $C_{0}$ used in the fits of Fig. 15. 


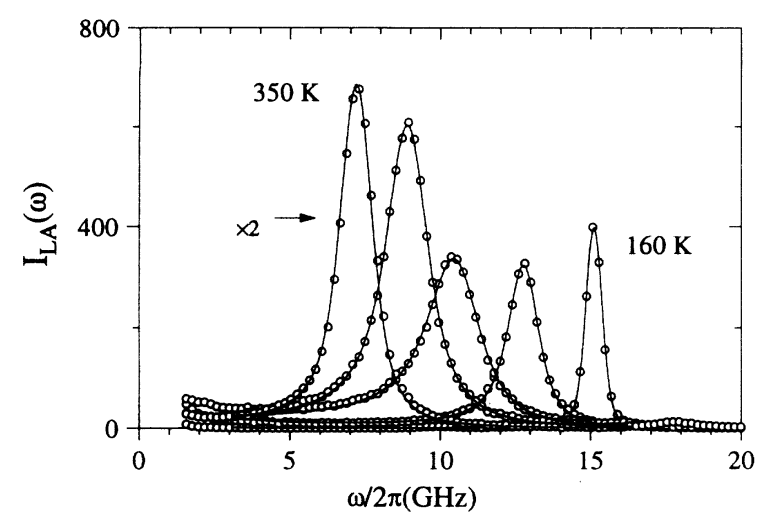

FIG. 15. Fits of $I_{\mathrm{LA}}$ spectra to the generalized hydrodynamics Eq. (6.3) with the Cole-Davidson model as memory function. $I_{0}, \Delta^{2}$, and $\tau_{\mathrm{CD}}$ are fitting parameters, with $\beta_{\mathrm{CD}}$ fixed at 0.68 . Temperatures are (from left to right): $350,280,240,200$, and $160 \mathrm{~K}$. ( $\mathrm{O})$ are experimental data, solid lines are the fitted results. For clarity only one-quarter of the experimental points are shown in the figure.

contributions to $m(\omega)$, will generally result in fit parameters of questionable physical significance. This is because the parameters used to model the $\alpha$ relaxation must be severely distorted to account for the $\beta$ relaxation as well [32]. But introducing additional parameters results in fits which may be even less reliable. One therefore seeks to represent $m(\omega)$ with as few adjustable parameters as possible.

In our Brillouin scattering study of CKN [32], we constructed an empirical memory function $m(\omega)$ containing both $\alpha$ - and $\beta$-relaxation contributions by identifying $m^{\prime \prime}(\omega)$ with the depolarized light-scattering spectrum. This empirical memory function led to a value of $T_{C}$ [from Eq. (6.1)] in good agreement with $T_{C}$ deduced from MCT fits to the depolarized spectra. Its use was based on the assumption that the structural relaxation dynamics probed by the LA mode are identical to the dynamics responsible for the depolarized light scattering. For CKN, this ansatz was justified by the observation that the $\alpha$ relaxation peak in the depolarized spectrum crosses the LA mode at the temperature where the LA mode linewidth is a maximum, so that $\tau_{\alpha}$ is about the same for

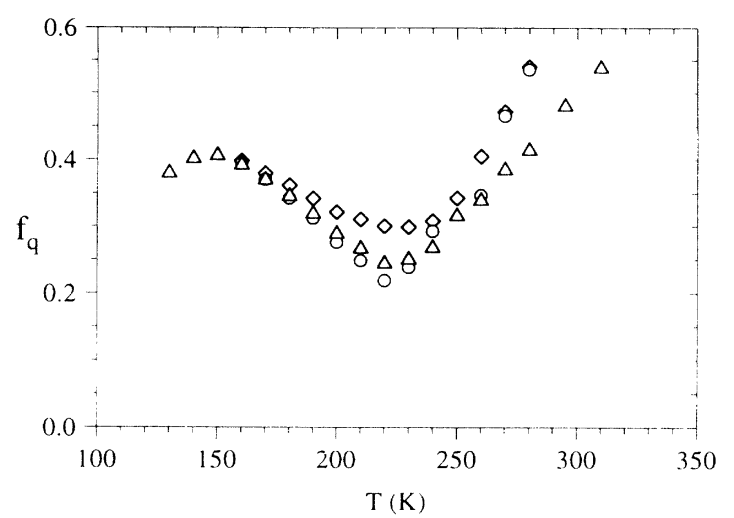

FIG. 16. Nonergodicity factors $f_{\mathrm{CD}}(\diamond), f_{B}(\bigcirc)$, and $f_{\gamma_{0}}(\triangle)$ deduced from fitting parameters found with the three different fitting methods [CD, hybrid with $B^{\prime \prime}$ free and $\gamma_{0}$ fixed, hybrid with $B^{\prime \prime}$ fixed and $\gamma_{0}(T)$ following a linear temperature dependence, respectively] with Eq. (6.1). The three results overlap on the low-temperature side. There are minima for all three around $220 \mathrm{~K}$.

both processes.

Figure 17 shows the temperature dependence of the LA mode peak frequency $\omega_{\mathrm{LA}}$, width $\Delta \omega_{\mathrm{LA}}$, and the peak frequency of $\alpha$ relaxation in the depolarized lightscattering spectrum, $\omega_{\max }$, for PC. The width of the LA mode is a maximum at $T \sim 240 \mathrm{~K}$, while the crossover of the LA mode peak frequency and the $\alpha$-relaxation peak frequency is at $T \sim 290 \mathrm{~K}$. At $240 \mathrm{~K}, \tau_{\alpha}$ (Brillouin) is about five times shorter than $\tau_{\alpha}$ of the depolarized spectrum. In a recent study of di-2-ethylhexyl phthalate, Floudas, Higgins, and Fytas [37] have similarly observed that the structural relaxation time inferred from the Brillouin linewidth is about one order of magnitude faster than that found from depolarized light-scattering or dielectric data. We have also observed a similar discrepancy for salol.

Since for PC the depolarized light-scattering spectrum and the LA mode seen in the Brillouin scattering spectrum therefore reflect different structural relaxation dynamics, the use of the empirical model for $m(\omega)$ cannot be justified for PC. We have therefore made a preliminary attempt to find $m(\omega)$ by combining the Cole-

TABLE III. Fit parameters for the LA mode with CD memory function.

\begin{tabular}{ccccccc}
\hline \hline$T(\mathrm{~K})$ & $\tau_{\mathrm{CD}}(\mathrm{ns})$ & $\tau_{K}(\mathrm{~ns})$ & $\Delta$ & $C_{0}(\mathrm{~cm} / \mathrm{s})$ & $C_{\infty}(\mathrm{cm} / \mathrm{s})$ & $f_{0}$ \\
\hline 160 & 4.03 & 2.50 & 91.3 & $1.95 \times 10^{5}$ & $2.52 \times 10^{5}$ & 0.398 \\
170 & 1.20 & 0.746 & 80.9 & $1.91 \times 10^{5}$ & $2.43 \times 10^{5}$ & 0.380 \\
180 & 0.412 & 0.256 & 71.7 & $1.88 \times 10^{5}$ & $2.35 \times 10^{5}$ & 0.362 \\
190 & 0.204 & 0.127 & 63.1 & $1.84 \times 10^{5}$ & $2.27 \times 10^{5}$ & 0.342 \\
200 & 0.102 & $6.36 \times 10^{-2}$ & 55.0 & $1.81 \times 10^{5}$ & $2.19 \times 10^{5}$ & 0.322 \\
210 & $5.85 \times 10^{-2}$ & $3.63 \times 10^{-2}$ & 49.9 & $1.77 \times 10^{5}$ & $2.13 \times 10^{5}$ & 0.311 \\
220 & $3.45 \times 10^{-2}$ & $2.15 \times 10^{-2}$ & 45.4 & $1.73 \times 10^{5}$ & $2.07 \times 10^{5}$ & 0.301 \\
230 & $2.32 \times 10^{-2}$ & $1.40 \times 10^{-2}$ & 43.0 & $1.70 \times 10^{5}$ & $2.03 \times 10^{5}$ & 0.299 \\
240 & $1.60 \times 10^{-2}$ & $9.92 \times 10^{-3}$ & 42.9 & $1.66 \times 10^{5}$ & $2.00 \times 10^{5}$ & 0.309 \\
250 & $1.15 \times 10^{-2}$ & $7.12 \times 10^{-3}$ & 47.7 & $1.63 \times 10^{5}$ & $2.01 \times 10^{5}$ & 0.343 \\
260 & $7.23 \times 10^{-3}$ & $4.48 \times 10^{-3}$ & 59.2 & $1.59 \times 10^{5}$ & $2.06 \times 10^{5}$ & 0.405 \\
270 & $5.06 \times 10^{-3}$ & $3.14 \times 10^{-3}$ & 73.9 & $1.55 \times 10^{5}$ & $2.14 \times 10^{5}$ & 0.472 \\
280 & $3.55 \times 10^{-3}$ & $2.20 \times 10^{-3}$ & 92.0 & $1.51 \times 10^{5}$ & $2.24 \times 10^{5}$ & 0.540 \\
\hline \hline
\end{tabular}




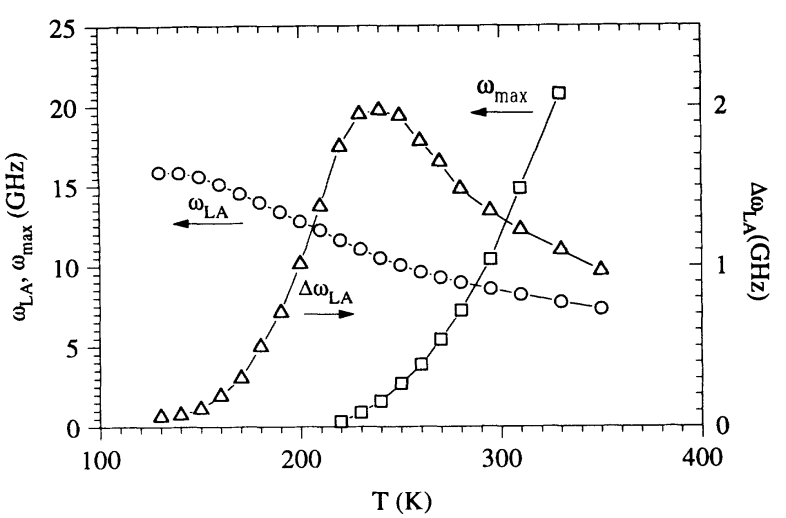

FIG. 17. Temperature dependence of the LA mode peak frequency $\omega_{\mathrm{LA}}(O)$ and linewidth $\Delta \omega_{\mathrm{LA}}(\Delta)$, and the $\alpha$-relaxation peak frequency $\omega_{\max }(\square)$ in the depolarized spectra of Fig. 7. The maximum width of the LA mode occurs at $T \sim 240 \mathrm{~K}$. At this temperature, the $\alpha$-peak frequency $\omega_{\max }$ is about five times smaller than the LA mode peak frequency.

Davidson expression for the $\alpha$ relaxation with the MCT prediction for the critical decay which is the highfrequency wing of the $\beta$-relaxation spectrum. The resulting hybrid model for $m(\omega)$ is

$$
m(\omega)=m_{\mathrm{CD}}(\omega)+B \omega^{a},
$$

where $B$ is a (complex) parameter and $m_{\mathrm{CD}}(\omega)$ is the $\mathrm{CD}$ function of Eq. (6.4). (The real and imaginary parts of $B$ are proportional to the cosine and sine transforms of $t^{-a}$.) Plots of Eq. (6.6) show a reasonable resemblance to the full $m(\omega)$ computed from MCT model calculations [38].

In Fig. 18 we plot $\tau$ values from several different experiments. $(O)$ are the $\tau_{\mathrm{CD}}$ from our CD model fits. $(\square)$ are the $\tau_{\alpha}$ results from the depolarized spectra. Note that the $\tau_{\mathrm{CD}}$ values fall below the $\tau_{\alpha}$ values and the discrepancy increases at low temperatures. The MCT predicts that while $\tau_{\alpha}$ for different processes may be different, all should follow a common temperature scale. We therefore fixed $\tau_{\mathrm{CD}}$ by taking the ratio of $\tau_{\alpha}$ to $\tau_{\mathrm{CD}}$ at $240 \mathrm{~K}$ where $\tau_{\mathrm{CD}}$ is set by $\omega_{\mathrm{LA}}$ and $\tau_{\alpha}$ is set by the position of the $\alpha$ peak $\omega_{\max }$, and then divided $\tau_{\alpha}$ at each temperature by this same ratio. The resulting $\tau_{C D}^{\prime}$ is shown in Fig. 18 by the $(\cdot-\cdot-)$ curve.

With this $\tau_{\mathrm{CD}}^{\prime}$, we carried out fits with the hybrid model with $\beta_{\mathrm{CD}}=0.68$, the same as in the pure CD fitting, and with $a=0.29$ obtained in the $\beta$-relaxation analysis in Sec. IV. The fitting parameters were $I_{0}, \Delta^{2}$, and $B^{\prime \prime}$. This hybrid model gave reasonable fits, but the temperature dependence of the resulting $\Delta^{2}$, as in the pure CD model analysis, still had an anomalous increase for temperatures higher than $\sim 230 \mathrm{~K}$. We also found that $\Delta^{2}$ and $B^{\prime \prime}$ are strongly correlated. Fitting with fixed $B^{\prime \prime}$ by constraining it to follow a linear temperature dependence with adjustable slope was also attempted, but reasonable fits for each spectrum in the whole temperature range could not be obtained. We also tried using fixed $B^{\prime \prime}$, allowing $\gamma_{0}$ to follow a linear temperature dependence. The fits were reasonable but $\Delta^{2}$ still had an anomalous temperature dependence as in the other cases.

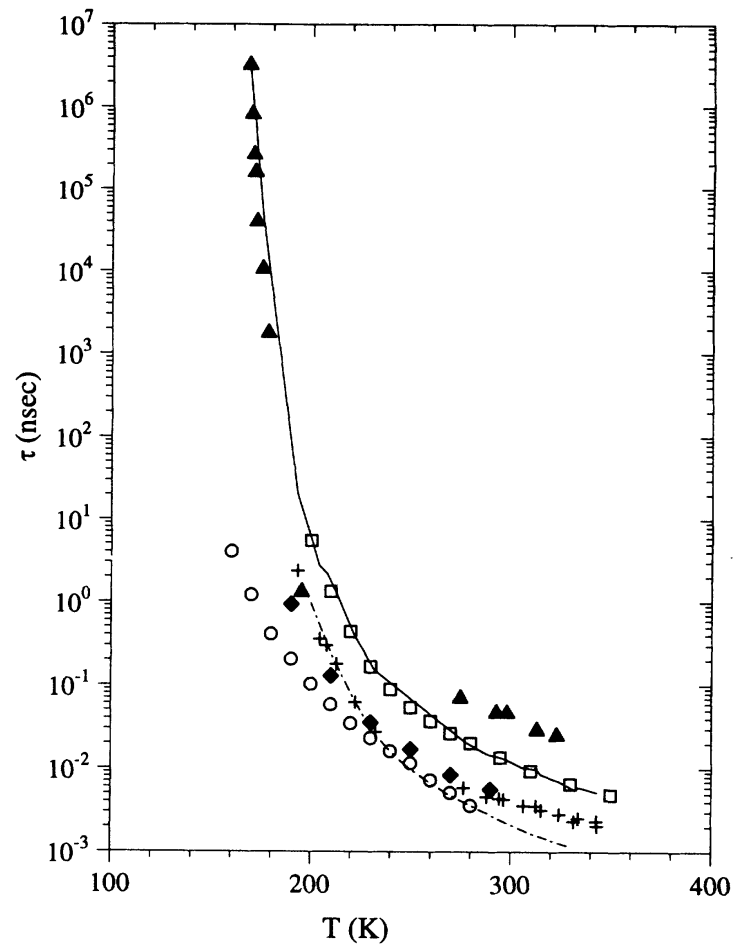

FIG. 18. Relaxation time $\tau_{\alpha}$ from various experiments: depolarized light scattering $(\square), \tau_{\mathrm{CD}}(O)$ from the fits of Fig. 14. The solid line is $\tau_{\eta}$ calculated from viscosity data [19] by using the Stokes-Einstein relation with $R=1.7 \AA$. (+) is $\tau_{\eta M}$ calculated from viscosity data using the Maxwell method. The $(\cdot-\cdot)$ line is the calculated $\tau_{C D}^{\prime}$ value used in the hybrid model fits discussed in the text. ( $\boldsymbol{\Delta})$ are results from dielectric measurements $[14,13,12,16]$. $(\$)$ are neutron scattering results [7].

Figure 16 shows the nonergodicity factors $f_{0}$ deduced from the fits via Eq. (6.1) for the three different analyses. There is no evidence for a cusp near $187 \mathrm{~K}$ which is expected to occur if Eq. (6.1) is valid. These results indicate that the hybrid model of Eq. (6.6) (or the constrained parameters employed in the fitting procedure) may not be adequate to describe $m(\omega)$. Although a more flexible model could be obtained by also allowing $\beta_{\mathrm{CD}}$ to vary freely, experience suggests that increasing the number of fitting parameters would produce fits of dubious significance. We therefore conclude that the determination of $T_{C}$ from Brillouin scattering experiments via Eq. (6.1) is not reliable unless the memory function can be independently determined.

\section{CONCLUSIONS}

Depolarized light-scattering studies of PC revealed a two-peak structure in the susceptibility spectrum $\chi^{\prime \prime}(\omega)$ similar to that previously reported for CKN [3], salol [4], and OTP [6]. Data analysis for $190 \leq T \leq 270 \mathrm{~K}$ based on the interpolation equation of the idealized MCT produced acceptable fits with an exponent parameter $\lambda=0.78$. Extrapolation of $\omega_{\min }^{2 a}$ and $\left(\chi_{\min }^{\prime \prime}\right)^{2}$ vs $T$ from these fits produced estimates of $T_{C}=(185 \pm 5) \mathrm{K}$. For $T<T_{C}$ the "knee" predicted by the idealized MCT was not observed so that pure scaling of the data, as for CKN 
[3], could not be carried out. We therefore employed an alternative scaling procedure, previously employed for salol [4], of constructing a MCT $\hat{\chi}_{+}^{\prime \prime}(\omega)$ master function for $\lambda=0.78$ and scaling the data onto the master curve. This procedure gave an estimate for $T_{C}$ consistent with the $T>T_{C}$ data.

An extended MCT analysis was also carried out for these data which quantitatively described the PC spectra. The extended analysis confirmed the values of $\lambda$ and $T_{C}$ found in the idealized MCT analysis. The results showed that for PC, the transition region separating the high- $T$ liquid regime from the low- $T$ glassy regime has a width of $\sim 10 \mathrm{~K}$, similar to salol but much smaller than CKN [5]. This result suggests that for molecular glass formers the buildup of hopping effects with decreasing $T$ may occur more rapidly than in molten salts, but more experiments are needed to see if this result is general.

Polarized Brillouin scattering experiments were performed in an attempt to elucidate the result reported by Elmroth, Borjesson, and Torell [10] in which $T_{C}$ found from a Brillouin scattering experiment was inconsistent with neutron scattering results. In our previous Brillouin scattering study of CKN [32] we had found that an empirical memory function could be constructed from the depolarized light-scattering spectrum which pro- duced good fits to the Brillouin scattering data and led to an estimate of $T_{C}$ in agreement with the value obtained from the depolarized light-scattering experiment. For PC, however, this procedure could not be employed because the primary relaxation time $\tau_{\alpha}$ of the structural relaxation process producing the depolarized lightscattering process is apparently about five times longer than that of the relaxation process coupling to the longitudinal acoustic modes. We attempted to fit the spectra with a hybrid memory function which would approximate the function predicted by MCT, but the results were inconclusive. At this point we tentatively conclude that the determination of $T_{C}$ from Brillouin scattering experiments is not generally reliable.

\section{ACKNOWLEDGMENTS}

We thank W. Götze for many discussions and suggestions, and C. A. Angell, C. Dreyfus, L. M. Torell, L. Borjesson, M. Elmroth, and K. A. Nelson for helpful discussions. Research at CUNY was supported by the U.S. National Science Foundation under Grant No. DMR9014344. M. Fuchs thanks the Deutsche Forschungsgemeinschaft for financial support under Contract No. Go 154/8-1.
[1] W. Götze, in Liquids, Freezing and the Glass Transition, edited by J. P. Hansen, D. Levesque, and J. Zinn-Justin (North-Holland, Amsterdam, 1990), p. 287.

[2] W. Götze and L. Sjögren, Rep. Prog. Phys. 55, 241 (1992).

[3] G. Li, W. M. Du, X. K. Chen, H. Z. Cummins, and N. J. Tao, Phys. Rev. A 45, 3867 (1992).

[4] G. Li, W. M. Du, A. Sakai, and H. Z. Cummins, Phys. Rev. A 46, 3343 (1992).

[5] H. Z. Cummins, W. M. Du, M. Fuchs, W. Götze, S. Hildebrand, A. Latz, G. Li, and N. J. Tao, Phys. Rev. E 47, 4223 (1993).

[6] W. Steffen, A. Patkowski, H. Glaser, G. Meier, and E. W. Fischer, Phys. Rev. E (to be published).

[7] L. Borjesson and W. S. Howells, J. Non-Cryst. Solids 131133, 53 (1991).

[8] L. Borjesson, M. Elmroth, and L. M. Torell, Chem. Phys. 149, 209 (1990)

[9] L. Borjesson, M. Elmroth, and L. M. Torell, J. Non-Cryst. Solids 131-133, 139 (1991).

[10] M. Elmroth, L. Borjesson, and L. M. Torell, Phys. Rev. Lett. 68, 79 (1992).

[11] A. K. M. Masood, R. A. Pethrick, A. J. Barlow, M. G. Kim, R. P. Plowiec, D. Barraclough, and J. A. Ladd, Adv. Mol. Relaxation Processes 9, 29 (1976).

[12] G. P. Johari and M. Goldstein, J. Chem. Phys. 53, 2372 (1970).

[13] J. R. Huck, G. A. Noyel, L. J. Jorat, and A. M. Bondeau, J. Electrostatics 12, 221 (1982).

[14] E. A. S. Cavell, J. Chem. Soc. Faraday Trans. 2 70, 78 (1974).

[15] J. Barthel, K. Bachhuber, R. Buchner, J. B. Gill, and M. Kleebauer, Chem. Phys. Lett. 167, 62 (1990).

[16] R. Payne and I. E. Theodorou, J. Phys. Chem. 76, 2892 (1972).
[17] L. J. Jorat, G. A. Noyel, and J. R. Huck, IEEE Trans. Electr. Insul. 26, 763 (1991).

[18] A. Schönhals, F. Kremer, A. Hofmann, E. W. Fischer, and E. Schlosser, Phys. Rev. Lett. 70, 3459 (1993).

[19] A. Bondeau and J. Huck, J. Phys (Paris) 46, 1717 (1985).

[20] C. A. Angell, J. Phys. Chem. Solids 49, 863 (1988).

[21] W. M. Du, G. Li, and H. Z. Cummins, Bull. Am. Phys. Soc. 37, 72 (1992).

[22] E. Leutheusser, Phys. Rev. A 29, 2765 (1984).

[23] U. Bengtzelius, W. Götze, and A. Sjölander, J. Phys. C 17, 5915 (1984)

[24] W. Götze, J. Phys. Condens. Matter 2, 8485 (1990).

[25] W. Götze and L. Sjögren, J. Phys. C 20, 879 (1987).

[26] M. Fuchs, W. Götze, I. Hofacker, and A. Latz, J. Phys. Condens. Matter 3, 5047 (1991).

[27] S. P. Das and G. F. Mazenko, Phys. Rev. A 34, 2265 (1986).

[28] W. Götze and L. Sjögren, Z. Phys. B 65, 415 (1987); J. Phys. C 21, 3407 (1988).

[29] M. Fuchs, W. Götze, S. Hildebrand, and A. Latz, J. Phys. Condens. Matter 4, 7709 (1992).

[30] M. Fuchs, W. Götze, and A. Latz, Chem. Phys. 149, 185 (1990).

[31] C. Dreyfus, M. J. Lebon, H. Z. Cummins, J. Toulouse, B. Bonello, and R. M. Pick, Phys. Rev. Lett. 69, 3666 (1992).

[32] G. Li, W. M. Du, J. Hernandez, and H. Z. Cummins, Phys. Rev. E 48, 1192 (1993).

[33] R. D. Mountain, J. Res. Natl. Bur. Stand. Sect. A 70, 207 (1966); 72, 95 (1968); Rev. Mod. Phys. 38, 205 (1966).

[34] N. J. Tao, G. Li, and H. Z. Cummins, Phys. Rev. B 43 , 5815 (1991).

[35] C. P. Lindsay and G. D. Patterson, J. Chem. Phys. 73, 3348 (1980).

[36] J. Toulouse and C. Launay, Rev. Sci. Instrum. 59, 492 
(1988).

[37] G. Floudas, J. S. Higgins, and G. Fytas, J. Chem. Phys. 96, 7672 (1992).

[38] H. Z. Cummins, G. Li, W. M. Du, and J. Hernandez, in
Proceedings of the 2nd International Meeting on Relaxations in Complex Systems, Alicante, Spain, June, 1993, edited by K. L. Ngai [J. Non-Cryst. Solids (to be published)]. 